\title{
A prediction method for plasma concentration by using a nonlinear grey Bernoulli combined model based on a self-memory algorithm
}

\author{
Xiaojun Guo ${ }^{a^{*}}$, Sifeng Liu ${ }^{b}$, Yingjie Yang ${ }^{c}$ \\ ${ }^{\text {a }}$ School of Science, Nantong University, Nantong 226019, China \\ ${ }^{\mathrm{b}}$ College of Economics and Management, Nanjing University of Aeronautics and Astronautics, Nanjing 211106, China \\ ${ }^{\mathrm{c}}$ Centre for Computational Intelligence, De Montfort University, Leicester, LE1 9BH, UK
}

\begin{abstract}
The goal of this work is to present and explore the application of a novel nonlinear grey Bernoulli combined model based on a self-memory algorithm, abbreviated as SA-NGBM, for modeling single-peaked sequences of time samples of acetylsalicylate plasma concentration following oral dosing. The self-memorization SA-NGBM routine reduces the dependence on a solitary initial value, as the initial state of the model utilizes multiple time samples. To test its forecasting performance, the SA-NGBM was used to extrapolate the plasma concentration predicted data, in comparison with the later time samples. The results were contrasted with those of the traditional optimized NGBM (ONGBM), exponential smoothing (ES) and simple moving average (SMA) using four popular accuracy and significance tests. That comparison showed that the SA-NGBM was much more accurate and efficient for matching the individual, nonlinear-system stochastic fluctuations than the existing ONGBM, ES and SMA models. The findings have potential applications for signal matching to similar small sample size, single-peaked, plasma concentration series.
\end{abstract}

\section{Keywords}

grey prediction theory; nonlinear grey Bernoulli model; self-memory algorithm; plasma concentration.

\section{Introduction}

With the rapid development of pharmaceutical science, practice indicates that the pharmacological action of many medicines is often closely related to their plasma concentration. The relationship of the therapeutic effect or toxicity of drugs follows plasma concentrations more closely than dosage. Plasma concentration refers to the total concentration in plasma after the drug is absorbed, and it usually can reflect the concentration variation of drug at the site of action. Therefore, the plasma concentration prediction for drugs is an important concern for modern medical research and has a great significance for rational drug use in patients and clinical medication guidance. The conventional prediction methods are pharmacokinetic models, constrained by kinetic parameters for individual drugs. For instance, in a compartment model, the organism could be assumed to be an abstract one-compartment, two-compartment or multicompartment dynamical model, according to different pharmic disposal speeds. Thus, the relationship between drug concentration and time in each compartment could be expressed by one differential equation, and the drug concentration could be obtained by means of solving the corresponding differential equations [1]. However, the traditional pharmacokinetic models should be performed under the hypothesis of many drug concentration data at multiple time points. Nonlinear iterative regression used to solve for the parameters easily leads to large computational demands and complicated processes. In recent years, some artificial intelligence methods, such as artificial neural networks (ANN), have been considered to forecast plasma concentrations [2]. On the premise of large sample data, these methods can perform prediction by means of training data without assuming a drug behavior model. However, it is difficult to acquire lots of high-quality clinical data due to many influencing factors and the absence of effective monitoring information. Meanwhile, the human body is an extremely complex organism that includes various random factors, so changes in plasma concentration in the body could be considered as an irregular and uncertain nonlinear system. Moreover, the grey systems theory is particularly suitable for studying those systems containing partially known information and

\footnotetext{
* Corresponding author. Tel.: +86 13862960631.
}

E-mail address: guoxj159@163.com (X. Guo). 
partially unknown information or underdetermined problems.

The traditional statistical prediction methods, such as the autoregressive moving-average model [3], the bilinear model [4] and the nonlinear autoregressive model [5], require assumptions about the system structure. However, there often exists uncertainty arising from the limitation of cognitive level and the influence of unknown disturbance. Hence, only part of the system structure could be fully realized, or it is very difficult to obtain the available information with large sample size. In view of the increasing complexity, uncertainty and chaos of the system's structure, several theories about uncertain systems have been produced, including fuzzy mathematics proposed by Zadeh [6] and the rough sets theory by Pawlak [7]. As an emerging branch of uncertainty systems science, the grey systems theory was initially proposed by Deng to study uncertain systems with small sample size and poor information [8,9]. Grey systems contain incomplete information which parts of the system are partially known or unknown. In this uncertainty theory, valuable information can be extracted by means of the accumulated generating operation (AGO) used on a small amount of historical observation (data). As an important theoretical component, the grey prediction models can weaken the randomness of original statistical data through AGO [10]. As a benefit over traditional statistical models, grey prediction models require only a limited amount of historical observation (data) and do not require knowing their statistical distribution. So far, they have already been effectively utilized in numerous fields, such as social economy [11,12], electric engineering [13], energy environment [14,15], transportation [16], public health [17,18,19] and have demonstrated satisfactory prediction results.

Among the grey systems, the $\operatorname{GM}(1,1)$ model is the basic core model and is especially useful for approximating homogenous exponential sequence [20]. Since first proposed by Deng, a series of improved grey prediction models were continuously developed for different types of time sequences, including the discrete grey model [21] and the $\operatorname{GM}(1,1)$ model with fractional order accumulation [22,23]. However, the original data distributions obtained from pharmacokinetic studies often present downward trends, such as from intravenous administration, or the single-peak types (an increase followed by a decrease), such as from extravascular administration. The conventional grey prediction models, as mentioned above, are inappropriate for the pharmacokinetic prediction. However, the nonlinear grey Bernoulli model termed $\operatorname{NGBM}(1,1)$ is a recently developed grey prediction model proposed by Chen, which is especially suitable for a class of approximate single-peak sequences $[24,25]$. After combining the $\operatorname{GM}(1,1)$ model with the Bernoulli differential equation, the power exponent in the NGBM can flexibly determine the trend curve of real systems and effectively manifest nonlinear characteristics. Nevertheless, certain time sequences of real systems, including nonlinear systems, often show large stochastic fluctuations due to uncertain external disturbances. Additionally, there is a limitation of these models that they are sensitive to the initial value of the original dynamic differential equation. It is inevitable that the original grey models will generate apparent modeling errors, so the self-memory algorithm could be considered for including the individual fluctuations occurring in general evolution tendencies.

Based on the irreversibility of natural and social phenomena, Cao first proposed the self-memory algorithm of dynamic systems by introducing the memory concept [26]. The history information should be investigated adequately if we want to realize the current status of system and predict its future trend. The self-memory algorithm (SA) exactly emphasizes the relationship between states before and after the current system status. It regards the environmental factors as external forcing, with a view to the evolution law of system per se. By introducing the memory function which contains historical information, the differential equation that restricts the dynamic system can be transformed into an appropriate difference-integral equation termed the self-memorization equation. It is a dynamical equation, though parameter estimation of the corresponding memory coefficients is a statistical method. Therefore, SA is the statistical-dynamic method to solve problems of nonlinear dynamic systems by integrating deterministic and random theories [27]. The predictive superiority of SA lies in that it not only avoids the drawback of using only one initial value in the initial-value problems of differential equations but also absorbs the advantage of extracting information from historical data in statistics. SA is a breakthrough for numerical solution of traditional initial-value problems and statistical methods, and it has been increasingly 
applied into various fields including meteorology, hydrology, and engineering science [28,29]. Meanwhile, SA was also combined with some grey prediction models including the $\operatorname{GM}(1,1)$ model, the $\operatorname{DHGM}(2,2)$ model and interval grey number models and showed satisfactory prediction results [30-32]. Accordingly, SA can be further introduced into the NGBM to predict the single-peaked nonlinear system together with individual stochastic fluctuations.

Aiming at the single-peaked nonlinear systems with small samples that exist in pharmacokinetic studies, this paper will construct a self-memory algorithm-based nonlinear grey Bernoulli model (SA-NGBM). The traditional NGBM can describe the integral single-peaked development tendencies of nonlinear systems, and the self-memory algorithm can capture the individual stochastic variations. The proposed SA-NGBM combines the prediction advantages of the SA and NGBM models through coupling the two prediction methods. The limitations of the traditional NGBM, such as sensitivity to the initial values of the differential equation, can be eliminated by using multi-timepoint initial field instead of an initial value from a single time point. Meanwhile, the SA-NGBM will be applied to simulate the plasma concentration of acetylsalicylic acid for method validation.

The remainder of this paper is organized as follows: A brief introduction to the general nonlinear grey Bernoulli model, the self-memory algorithm and the self-memory algorithm-based nonlinear grey Bernoulli model are presented and discussed in Section 2. In Section 3, an empirical analysis on plasma concentration of acetylsalicylic acid are adopted to verify the feasibility and effectiveness of the novel SA-NGBM proposed here. Finally, some conclusions are drawn and future work proposed in Section 4.

\section{Methods}

\subsection{General nonlinear grey Bernoulli model (NGBM)}

In grey systems theory, the NGBM is a first-order single-variable grey model with an interpolated coefficient in the background value. The basic form and characteristics of general NGBM can be summarized as follows [24]:

First, assume that the original historical time sequence

$$
F_{t}^{0}=\left\{f_{t}^{0} \mid t \in 1,2, \cdots, n\right\}
$$

is a nonnegative data sequence, where the observational data $f_{t}^{0}$ corresponds to the system input at time $t$ and $n$ is the total number of time points. Set $f_{1}^{1}=f_{1}^{0}$ and construct $F_{t}^{1}$ by a one-time accumulated generation operation (1-AGO), which is given by

$$
F_{t}^{1}=\left\{f_{t}^{1} \mid t \in 1,2, \cdots, n\right\},
$$

where $f_{t}^{1}=\sum_{k=1}^{t} f_{k}^{0}, t=1,2, \cdots, n$ and $F_{t}^{1}$ is termed as the 1-AGO sequence of $F_{t}^{0}$.

Next, based on the principle of elementary differential equations, the Bernoulli equation [33] is introduced to establish the nonlinear grey Bernoulli equation for the NGBM as the following form

$$
f_{t}^{0}+a g_{t}{ }^{1}=b\left(g_{t}\right)^{\gamma}, t=2,3, \cdots, n,
$$

where $f_{t}^{0}$ is called a grey derivative, $g_{t}^{1}=\phi \cdot f_{t}^{1}+(1-\phi) \cdot f_{t-1}^{1}$ is referred to as the background value of the grey derivative $f_{t}^{0}$, and $\phi$ is called the interpolated coefficient of the background value within a closed interval $[0,1]$, which is traditionally set to 0.5 . When $\phi$ is equal to 0.5 , Eq. (3) is the general NGBM [24]. When $\phi$ is an indefinite value in the interval [0,1], Eq. (3) is the Nash nonlinear grey Bernoulli model (NNGBM) [25].

Then, at sampling time $\Delta t=1$, the parameters $a$ and $b$ in matrix $\hat{R}$ can be obtained through least-squares estimation according to Eq. (3) as follows:

$$
\hat{R}=\left(\begin{array}{l}
\hat{a} \\
\hat{b}
\end{array}\right)=\left(B^{\mathrm{T}} B\right)^{-1} B^{\mathrm{T}} F_{n},
$$


where $B=\left[\begin{array}{cc}-g_{2}^{1} & \left(g_{2}^{1}\right)^{\gamma} \\ -g_{3}^{1} & \left(g_{3}^{1}\right)^{\gamma} \\ \vdots & \vdots \\ -g_{n}^{1} & \left(g_{n}^{1}\right)^{\gamma}\end{array}\right]$ and $F_{n}=\left[\begin{array}{c}f_{2}^{0} \\ f_{3}^{0} \\ \vdots \\ f_{n}^{0}\end{array}\right]$. On the basis of the monotonically increasing sequence $F_{t}^{1}$, the whitenization differential equation of the NGBM (i.e., a first-order differential equation) can be established as follows:

$$
\frac{\mathrm{d} F_{t}^{1}}{\mathrm{~d} t}+a F_{t}^{1}=b\left(F_{t}^{1}\right)^{\gamma}
$$

where the parameters $a$ and $b$ are called the developing and grey input coefficients, respectively, and the adjustable parameter $\gamma \neq 1$ is called the power exponent.

Thus, Eq. (5) can be solved together with initial value $\hat{f}_{1}^{1}=f_{1}^{0}$, and the corresponding particular solution is

$$
\hat{f}_{t+1}^{1}=\left[\left(\left(f_{1}^{0}\right)^{1-\gamma}-\frac{b}{a}\right) e^{-a(1-\gamma) t}+\frac{b}{a}\right]^{1 /(1-\gamma)}, \gamma \neq 1, \forall t \geq 1,
$$

which is called the time-response sequence of the NGBM. Then, the system output $\hat{f}_{t+1}^{1}$ of dependent variable $F_{t}^{1}$ can be obtained from Eq. (6), accordingly. Application of the first-order inverse accumulated generation operation (1-IAGO) to $\hat{f}_{t+1}^{1}$ allows the simulation and forecasting values of the original sequence $F_{t}^{0}$ to be obtained as follows:

$$
\hat{f}_{t+1}^{0}=\hat{f}_{t+1}^{1}-\hat{f}_{t}, \quad \forall t \geq 1 .
$$

Remark. The NGBM is a sort of significant grey prediction model especially useful for the nonlinear small-sample time sequences, where the adjustable power exponent $\gamma$ plays an important role. The adjustable value of $\gamma$ that matches with actual data could be acquired by certain optimization techniques. When $\gamma=0$, the NGBM will degenerate into the traditional $\operatorname{GM}(1,1)$ model; when $\gamma=2$, the NGBM will degenerate into the grey Verhulst model. Comparatively, the adjustable power exponent $\gamma$ in the NGBM can flexibly fit the actual historical observation (data) and effectively reflect the nonlinear characteristics of real systems. Numerous correlational studies indicate that the NGBM is ideally suitable for nonlinear systems including a class of approximate single-peak sequences [18,24,25,34-37].

Nevertheless, there are large stochastic fluctuation phenomena inevitably present in those real nonlinear systems due to uncertain external disturbances. The general NGBM cannot reveal the individual stochastic fluctuations but can only describe the integral single-peaked evolution tendency. Under such circumstances, it is impossible to ignore the apparent modeling errors by using the general NGBM. Therefore, it is very essential for exploring how to detect the individual stochastic fluctuations present in general evolution tendency of nonlinear systems with small samples.

\subsection{The self-memory algorithm}

Chaotic dynamics indicate that there are random behaviors exhibited even in simple dynamic systems due to nonlinearities; it also implies that a prediction method must consider randomness along with dynamics. The self-memory algorithm (SA) of dynamic systems has exactly filled the gap between determinism and randomness theory and has raised questions about the predictability of systems. As system change processes are often irreversible, SA was developed by introducing the memory function, which contains historical information, into the system dynamic equation. After defining the inner product in Hilbert space, the system dynamic equation (i.e., a differential equation) can be transformed into an appropriate difference-integral equation, which is termed as a self-memorization one. Then, the self-memorization equation can be used to predict the system status by means of 
discretization and estimating the memory coefficients. The original dynamic differential equation has the weakness of being sensitive to the initial value. Comparatively, the self-memorization equation can eliminate this drawback on account that it contains initial fields for multiple time points instead of initial fields at only a single time point. Accordingly, the prediction superiority of SA lies in that it not only merges dynamic calculations together with parameter estimation from historical data but also adequately extracts historical information from real systems in statistics.

The evolution process of system maybe described by a nonlinear function as follows:

$$
x(t+\Delta t)=f(\lambda, x(t)),
$$

where $x$ is a state variable in relation to time $t$, and $\lambda$ represents a set of all control parameters. Generally, $t_{0}, t_{1}, t_{2}, \cdots$ denote various moments, and $x_{0}, x_{1}, x_{2}, \cdots$ denote corresponding states. Let

$$
x_{n} \equiv x\left(t_{n}\right), t_{n}=t_{0}+n \Delta t,
$$

then Eq. (8) can be transformed into $x_{n+1}=f\left(\lambda, t, x_{n}\right)$. There are various kinds of external disturbances that result in system uncertainty. After adding a stochastic term that affects the system state, the system equation can be expressed as follows:

$$
\frac{\mathrm{d} x}{\mathrm{~d} t}=f(\lambda, x(t))+g(x) Q(t)
$$

where $g(x) Q(t)$ is multiplicative noise, $g Q(t)$ is additive noise and $g$ is a constant.

Assume that one time set $T=\left[t_{-p}, t_{-p+1}, \cdots, t_{-1}, t_{0}, t_{1}, \cdots, t_{q-1}, t_{q}\right]$, where $t_{-p}, t_{-p+1}, \cdots, t_{-1}$ denote historical time points (observation points) from $t_{-p}$ to $t_{-1}, t_{0}$ denotes the current initial time point (observation point), $t_{1}, \cdots, t_{q-1}, t_{q}$ denote the future forecasting time points (observation points) from $t_{1}$ to $t_{q}$. The spatial set is expressed as $R=\left[r_{\alpha}, \cdots, r_{i}, \cdots, r_{\beta}\right]$, where $r_{i}$ is the spatial point which is inspected. The inner product space $L^{2}: T \times R$, which is in the Hilbert space, can be defined as follows:

$$
(f, g)=\int_{a_{0}}^{b_{0}} f(\xi) g(\xi) \mathrm{d} \xi,\left(f, g \in L^{2}\right) .
$$

Generally, the system dynamics equation can be expressed as follows:

$$
\frac{\mathrm{d} x}{\mathrm{~d} t}=F(x, \lambda, t)
$$

where $x$ is a variable, $\lambda$ is a parameter, $t$ is time interval series, and $F(x, \lambda, t)$ is termed the dynamic kernel. Eq. (12) reflects the relationship between local change of $x$ at time $t$ and source function $F$. Then, the memory function is defined as $\beta(r, t)$, where $\beta$ is the function of $r$ and $t$, and $|\beta(r, t)| \leq 1$. If it is limited to spatial point $r_{i}$, the memory function can be simplified to $\beta(t)$.

The ordinary initial-value problem in the general NGBM corresponds to a Markov process where the next value depends only on the present value. While we make a prediction by SA, the memory function $\beta(t)$ possesses functions in memorizing $p+1$ values of $x$, because $p$ values before $t_{0}$ are always utilized. This is the algorithm mechanism for introducing a memory function $\beta(t)$ into the novel SA-NGBM.

\subsection{Self-memory algorithm-based nonlinear grey Bernoulli model (SA-NGBM)}

Based on the algorithms mentioned above, the SA prediction method is coupled with the general NGBM to construct a novel SA-NGBM. The modeling flowsheet of the SA-NGBM is displayed in Figure 1. The calculation was performed with the aid of the MATLAB program in order to decrease the computational complexity for model parameters, memory coefficients, and so on. 


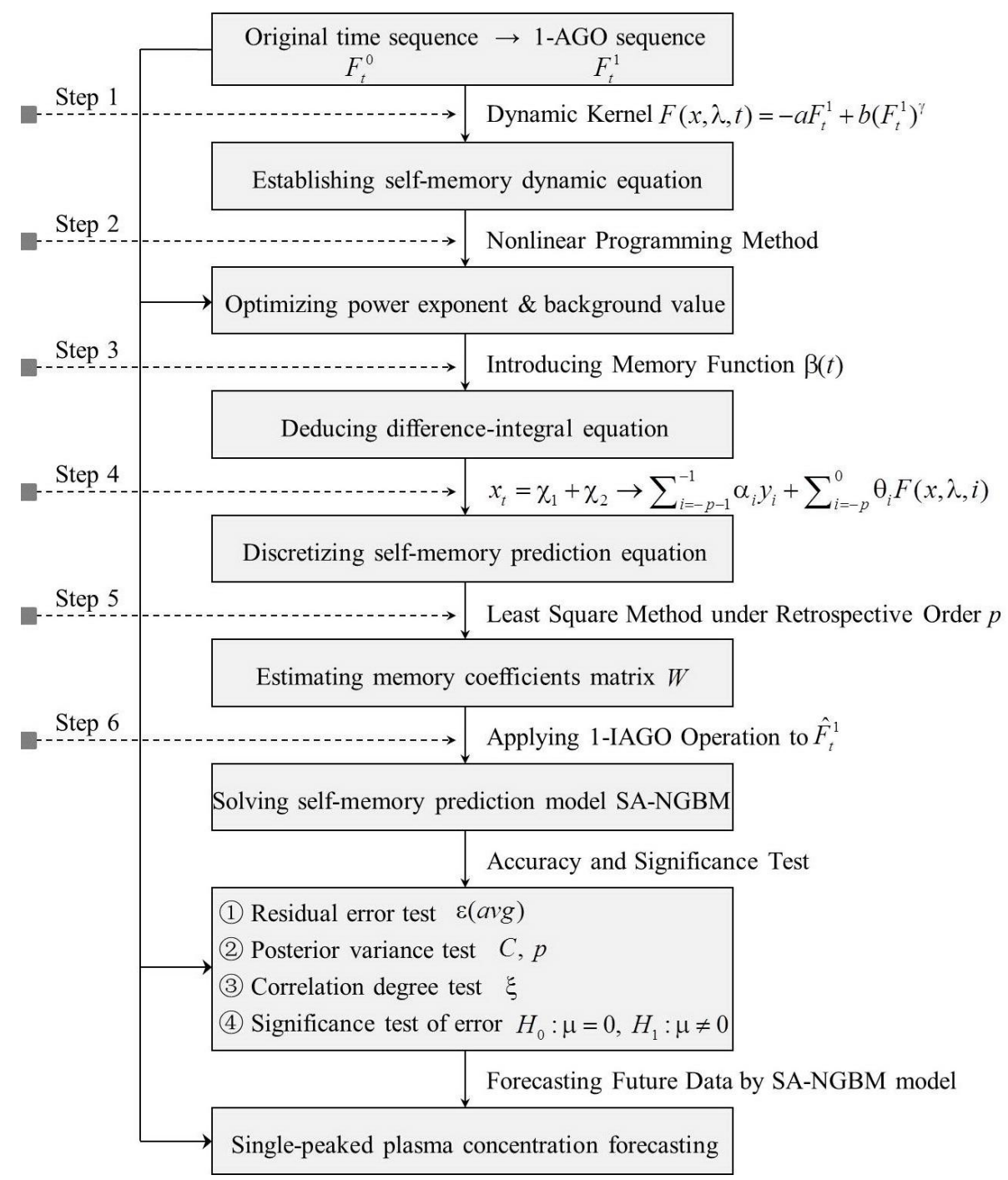

FIGURE 1 The modeling flowsheet of the SA-NGBM.

Considering nonlinear systems with small sample sizes, it is assumed that the original time sequence and the 1-AGO sequence are $F_{t}^{0}=\left\{f_{t}^{0} \mid t \in 1,2, \cdots, n\right\}$ and $F_{t}^{1}=\left\{f_{t}^{1} \mid t \in 1,2, \cdots, n\right\}$ respectively. Then, the coupling modeling procedure of the SA-NGBM is described as follows.

Step 1: Establishing the self-memory dynamic equation.

Let the differential equation $\frac{\mathrm{d} F_{t}^{1}}{\mathrm{~d} t}=-a F_{t}^{1}+b\left(F_{t}^{1}\right)^{\gamma}$, which is equal to the whitenization equation (5) of the NGBM, be $F(x, \lambda, t)=-a F_{t}^{1}+b\left(F_{t}^{1}\right)^{\gamma}$. Then, the self-memory dynamic equation of the novel SA-NGBM it is considered to be in the form $\frac{\mathrm{d} x}{\mathrm{~d} t}=F(x, \lambda, t)$, where the source function of dynamic kernel $F(x, \lambda, t)=-a F_{t}^{1}+b\left(F_{t}^{1}\right)^{\gamma}$.

Step 2: Optimizing the power exponent and background value.

The superiority of the NGBM lies in the fact that the adjustable value of power exponent $\gamma$, which matches with actual data, could be acquired by certain optimization techniques. Optimization occurs by supposing that the objective function is the minimization of mean absolute percentage error (MAPE) and that the constraint condition is the relationship among parameters $a, b$ and $\gamma$ [35-37]: 


$$
\begin{aligned}
& \underset{\gamma, \phi}{\operatorname{Min}} \operatorname{MAPE}=\frac{1}{n-1} \sum_{k=2}^{n}\left|\frac{\hat{f}_{t}^{0}-f_{t}^{0}}{f_{t}^{0}}\right| \\
& \text { S.T. }\left\{\begin{array}{l}
\gamma \neq 1 \\
0 \leq \phi \leq 1 \\
\hat{f}_{t}^{0}=\hat{f}_{t}^{1}-\hat{f}_{t-1}^{1} \\
\hat{f}_{t}^{1}=\left\{d+\left[\left(f_{1}^{0}\right)^{1-\gamma}-d\right] e^{-a(1-\gamma) t}\right\}^{1 / 1-\gamma} \\
d=\frac{\sum_{k=2}^{n}\left(g_{t}^{1}\right)^{2} \cdot \sum_{k=2}^{n} f_{t}^{0}\left(g_{t}^{1}\right)^{\gamma}-\sum_{k=2}^{n}\left(g_{t}^{1}\right)^{\gamma+1} \cdot \sum_{k=2}^{n} f_{t}^{0} g_{t}^{1}}{\sum_{k=2}^{n}\left(g_{t}^{1}\right)^{\gamma+1} \cdot \sum_{k=2}^{n} f_{t}^{0}\left(g_{t}^{1}\right)^{\gamma}-\sum_{k=2}^{n}\left(g_{t}^{1}\right)^{2 \gamma} \cdot \sum_{k=2}^{n} f_{t}^{0} g_{t}^{1}} \\
g_{t}^{1}=\phi \cdot f_{t}^{1}+(1-\phi) \cdot f_{t-1}^{1} \\
t=2,3, \cdots, n
\end{array}\right.
\end{aligned}
$$

The adjustable value of the power exponent $\gamma$ can be optimized by a nonlinear programming model (13). Meanwhile, the appropriate interpolated coefficient $\phi$, assumed to have a closed interval $[0,1]$, can be optimized against the background value $g_{t}^{1}=\phi \cdot f_{t}^{1}+(1-\phi) \cdot f_{t-1}^{1}$. This equation can be conveniently solved with the aid of LINGO software or MATLAB software, thereby obtaining the optimal power exponent $\gamma$ and the interpolated coefficient $\phi$.

Step 3: Deducing the difference-integral equation.

Assume that one time set $T=\left[t_{-p}, t_{-p+1}, \cdots, t_{-1}, t_{0}, t_{1}, \cdots, t_{q-1}, t_{q}\right]$, where the number $p$ of historical time points is the retrospective order of the system, and $q$ is the number of future forecasting time points. If considering the multiple historical time points $t_{-p}, t_{-p+1}, \cdots, t_{-1}$ and a single forecasting time point $t$, the memory function $\beta(t)$ and inner product operation (11) are introduced into $\frac{\mathrm{d} x}{\mathrm{~d} t}=-a F_{t}^{1}+b\left(F_{t}^{1}\right)^{\gamma}$. Meanwhile, the variable $x$ and memory function $\beta(t)$ are supposed to meet the conditions of continuous, differentiable and integrable. Then, the system dynamic equation can be changed to an analytic formula as follows:

$$
\int_{t_{-p}}^{t} \beta(\tau) \frac{\partial x}{\partial \tau} \mathrm{d} \tau=\int_{t_{-p}}^{t} \beta(\tau)\left[-a F_{\tau}^{1}+b\left(F_{\tau}^{1}\right)^{\gamma}\right] \mathrm{d} \tau
$$

which can be regarded as the weighted integral whose weight is memory coefficient $\beta(t)$. Eq. (14) then becomes

$$
\int_{t_{-p}}^{t_{-p+1}} \beta(\tau) \frac{\partial x}{\partial \tau} \mathrm{d} \tau+\int_{t_{-p+1}}^{t_{-p+2}} \beta(\tau) \frac{\partial x}{\partial \tau} \mathrm{d} \tau+\cdots+\int_{t_{0}}^{t} \beta(\tau) \frac{\partial x}{\partial \tau} \mathrm{d} \tau=\int_{t_{-p}}^{t} \beta(\tau)\left[-a F_{\tau}^{1}+b\left(F_{\tau}^{1}\right)^{\gamma}\right] \mathrm{d} \tau .
$$

The method of integration by parts and the mean value theorem of integrals are applied to all integral terms on the left-hand side of Eq. (15). After merging and eliminating the similar terms, a difference-integral equation is deduced as:

$$
\beta_{t} x_{t}-\beta_{-p} x_{-p}-\sum_{i=-p}^{0} x_{i}^{m}\left(\beta_{i+1}-\beta_{i}\right)-\int_{t_{-p}}^{t} \beta(\tau)\left[-a F_{\tau}^{1}+b\left(F_{\tau}^{1}\right)^{\gamma}\right] \mathrm{d} \tau=0
$$

where $\beta_{t} \equiv \beta(t), \quad x_{t} \equiv x(t), \quad \beta_{i} \equiv \beta\left(t_{i}\right), \quad x_{i} \equiv x\left(t_{i}\right), \quad i=-p,-p+1, \cdots, 0, \quad$ and mid-value $x_{i}^{m} \equiv x\left(t_{m}\right)$, $t_{i}<t_{m}<t_{i+1}$.

Step 4: Discretizing the self-memory prediction equation.

Let $x_{-p} \equiv x_{-p-1}^{m}$ and $\beta_{-p-1} \equiv 0$. Eq. (16) can then be transformed into

$$
x_{t}=\frac{1}{\beta_{t}} \sum_{i=-p-1}^{0} x_{i}^{m}\left(\beta_{i+1}-\beta_{i}\right)+\frac{1}{\beta_{t}} \int_{t_{-p}}^{t} \beta(\tau)\left[-a F_{\tau}^{1}+b\left(F_{\tau}^{1}\right)^{\gamma}\right] \mathrm{d} \tau=\chi_{1}+\chi_{2},
$$


and is termed the $p$-order self-memory prediction equation, where $p$ denotes the retrospective order. The self-memory equation (17) emphasizes that the predicted value $x_{t}$ at time point $t$ is the joint contribution of self-memory term $\chi_{1}$ and exogenous effect term $\chi_{2}$. Eq. (17) can be discretized by replacing the integral operation with summation, replacing the differential with a difference, and replacing the mid-value $x_{i}^{m}$ with the mean value at two contiguous time points, namely, $x_{i}^{m}=\frac{1}{2}\left(x_{i+1}+x_{i}\right) \equiv y_{i}$. The self-memory equation (17) can be discretized by taking the equidistance time interval $\Delta t_{i}=t_{i+1}-t_{i}=1$ for the time points, and merging $\beta_{t}$ with $\beta_{i}$ :

$$
x_{t}=\sum_{i=-p-1}^{-1} \alpha_{i} y_{i}+\sum_{i=-p}^{0} \theta_{i}\left[-a F_{i}^{1}+b\left(F_{i}^{1}\right)^{\gamma}\right]
$$

where $\alpha_{i}=\left(\beta_{i+1}-\beta_{i}\right) / \beta_{t}$ and $\theta_{i}=\beta_{i} / \beta_{t}$ are called memory coefficients.

Step 5: Estimating the memory coefficients matrix.

When compared to a general differential equation, there is an extra memory function $\beta(t)$ present in the self-memory equation. Therefore, the self-memory equation has no analytic solution, and the numerical solution only can be solved with the aid of a computer. If regarding $F$ and $x$ as the input and output of the systems respectively, the self-memory equation can be solved by using the ordinary least squares method (OLS). Assume that there is a historical data sequence at $L(L>p)$ time points, and OLS is used to estimate the memory coefficients $\alpha_{i}$ and $\theta_{i}$ in Eq. (18). Let

$$
\begin{aligned}
\underset{L \times 1}{X_{t}}=\left[\begin{array}{c}
x_{t 1} \\
x_{t 2} \\
\vdots \\
x_{t L}
\end{array}\right], \underset{L \times(p+1)}{Y}=\left[\begin{array}{cccc}
y_{-p-1,1} & y_{-p, 1} & \cdots & y_{-1,1} \\
y_{-p-1,2} & y_{-p, 2} & \cdots & y_{-1,2} \\
\vdots & \vdots & \ddots & \vdots \\
y_{-p-1, L} & y_{-p, L} & \cdots & y_{-1, L}
\end{array}\right], \quad \underset{(p+1) \times 1}{A}=\left[\begin{array}{c}
\alpha_{-p-1} \\
\alpha_{-p} \\
\vdots \\
\alpha_{-1}
\end{array}\right], \\
\underset{L \times(p+1)}{\Gamma}=\left[\begin{array}{cccc}
\left(-a F_{-p}^{1}+b\left(F_{-p}^{1}\right)^{\gamma}\right)_{1} & \left(-a F_{-p+1}^{1}+b\left(F_{-p+1}^{1}\right)^{\gamma}\right)_{1} & \cdots & \left(-a F_{0}^{1}+b\left(F_{0}^{1}\right)^{\gamma}\right)_{1} \\
\left(-a F_{-p}^{1}+b\left(F_{-p}^{1}\right)^{\gamma}\right)_{2} & \left(-a F_{-p+1}^{1}+b\left(F_{-p+1}^{1}\right)^{\gamma}\right)_{2} & \cdots & \left(-a F_{0}^{1}+b\left(F_{0}^{1}\right)^{\gamma}\right)_{2} \\
\vdots & \vdots & \ddots & \vdots \\
\left(-a F_{-p}^{1}+b\left(F_{-p}^{1}\right)^{\gamma}\right)_{L} & \left(-a F_{-p+1}^{1}+b\left(F_{-p+1}^{1}\right)^{\gamma}\right)_{L} & \cdots & \left(-a F_{0}^{1}+b\left(F_{0}^{1}\right)^{\gamma}\right)_{L}
\end{array}\right], \underset{(p+1) \times 1}{\Theta}=\left[\begin{array}{c}
\theta_{-p} \\
\theta_{-p+1} \\
\vdots \\
\theta_{0}
\end{array}\right],
\end{aligned}
$$

then the matrix form of Eq. (18) can be expressed as follows:

$$
X_{t}=Y A+\Gamma \Theta .
$$

If we let $Z=[Y, \Gamma], W=\left[\begin{array}{c}A \\ \Theta\end{array}\right]$, then Eq. (19) turns into $X_{t}=Z W$, and can thereby be described as the least square estimation of memory coefficients matrix $W$ is obtained by OLS:

$$
W=\left(Z^{\mathrm{T}} Z\right)^{-1} Z^{\mathrm{T}} X_{t}
$$

Step 6: Solving the self-memory prediction model.

After the memory coefficient matrix $W$ is substituted into the discretized prediction equation (18), the simulation and forecasting value $\hat{f}_{t}^{1}(\forall t \geq 1)$ of the 1-AGO sequence $F_{t}^{1}$ can be acquired. Then, by applying the 1-IAGO to $\hat{f}_{t}^{1}$, its corresponding inverse accumulated value $\hat{f}_{t}^{0}(\forall t \geq 1)$ can be solved as follows:

$$
\hat{f}_{t}^{0}=\hat{f}_{t}^{1}-\hat{f_{-1}^{1}}, \quad \forall t \geq 1,
$$

where $\hat{f}_{0}^{1} \equiv 0$. Consequently, the simulation and forecasting sequence $\hat{F}_{t}^{0}=\left\{\hat{f}_{t}^{0} \mid \forall t \geq 1\right\}$ of the original 
sequence $F_{t}^{0}$ is obtained.

\subsection{Accuracy and significance test of forecasting performance}

The purpose of prediction to provide services for decision making, and the credible and accurate predicting of outcomes are the precondition for scientific decision. Therefore, the accuracy and significance test results are important evaluation criteria for comparing the SA-NGBM with other prediction models. Only after the prediction model has passed through the accuracy and significance tests can it be used for extrapolation and application. In this study, four popular test indices for grey prediction methods have been used to compare the accuracy of different prediction models and test the corresponding significance as follows $[9,20]$.

Let $f_{t}^{0}(t \in 1,2, \cdots, n)$ denote the actual values at time point $t$ of the original sequence $F_{t}^{0}$, and $\hat{f}_{t}^{0}(t \in 1,2, \cdots, n)$ denote the corresponding predicted values. Thus $q(t)=f_{t}^{0}-\hat{f}_{t}^{0}(t \in 1,2, \cdots, n)$ is called the residual error at specific time point $t$. In addition, the RMSE $=\sqrt{\frac{1}{n} \sum_{t=1}^{n}\left(f_{t}^{0}-\hat{f}_{t}^{0}\right)^{2}}$ denotes the root mean square error.

Test criterion 1: Residual error test

The residual error test is one type of pointwise test method. The relative percentage error $\mathrm{RPE}_{t}$ at time point $t$ is defined as

$$
\operatorname{RPE}_{t}=\varepsilon(t)=\frac{q(t)}{f_{t}^{0}} \times 100 \%, t \in 2,3, \cdots, n,
$$

where $q(t)$ denotes the residual error at time point $t$. The average relative percentage error ARPE at all time points is expressed as

$$
\mathrm{ARPE}=\varepsilon(\operatorname{avg})=\frac{1}{n-1} \sum_{t=2}^{n}|\varepsilon(t)|, \quad t \in 2,3, \cdots, n .
$$

For a given $\alpha>0$, when $\mathrm{ARPE}<\alpha$ and $\mathrm{RPE}_{t}<\alpha$, then the model is supposed to pass the residual error test.

Test criterion 2: Posterior variance test

Let $\bar{f}=\frac{1}{n} \sum_{t=1}^{n} f_{t}^{0}$ and $S_{1}^{2}=\frac{1}{n} \sum_{t=1}^{n}\left(f_{t}^{0}-\bar{f}\right)^{2}$ denote the mean value and variance of the original sequence $F_{t}^{0}$, respectively. Moreover, $\bar{q}=\frac{1}{n} \sum_{t=1}^{n} q(t)$ and $S_{2}{ }^{2}=\frac{1}{n} \sum_{t=1}^{n}(q(t)-\bar{q})^{2}$ denote the mean value and variance of the residual error sequence $Q^{0}$, respectively. The ratio of mean square error $C=S_{2} / S_{1}$ is termed as the posterior variance ratio. For a given $C_{0}>0$, if there is $C<C_{0}$, then the model is a posterior-variance-ratio-qualified model. In the same way, for a given $p_{0}>0$, if there is $p=P\left\{|q(t)-\bar{q}|<0.6745 S_{1}\right\}>p_{0}$, then the model is a small-error-probability-qualified model. Accordingly, the model is considered to pass the posterior variance test.

Test criterion 3: Correlation degree test

Let $\xi=\frac{1+|\delta|+|\hat{\delta}|}{1+|\delta|+|\hat{\delta}|+|\hat{\delta}-\delta|}$ denote the absolute correlation degree between the original sequence $F_{t}^{0}$ and predicted sequence $\quad \hat{F}_{t}^{0} \quad, \quad$ where $\quad|\delta|=\left|\sum_{t=2}^{n-1} f_{t}^{0}+\frac{1}{2} f_{n}^{0}\right| \quad, \quad|\hat{\delta}|=\left|\sum_{t=2}^{n-1} \hat{f}_{t}^{0}+\frac{1}{2} \hat{f}_{n}^{0}\right| \quad$ and $|\hat{\delta}-\delta|=\left|\sum_{t=2}^{n-1}\left(\hat{f}_{t}^{0}-f_{t}^{0}\right)+\frac{1}{2}\left(\hat{f}_{n}^{0}-f_{n}^{0}\right)\right|$. For a given $\xi_{0}>0$, if the model satisfies $\xi_{>} \xi_{0}$, then it is regarded to 
pass the correlation degree test.

Test criterion 4: Significance test of error

Let $E_{t}=\frac{q(t)}{f_{t}^{0}}(t \in 2,3, \cdots, n)$, and it is generally considered to obey a normal distribution, i.e., $E_{t} \sim N\left(\mu, \sigma^{2}\right)$, where $\mu$ and $\sigma^{2}$ are unknown. The higher the prediction accuracy is, the closer to zero the mathematical expectation of relative error obeying a normal distribution is. Therefore, the level of prediction accuracy is equivalent to the hypothesis testing as follows:

$$
H_{0}: \mu=0, H_{1}: \mu \neq 0 \text {. }
$$

For a given confidence level $\alpha$, the test statistic $t(n-1)=\frac{\bar{E}}{s / \sqrt{n}}$ is constructed. If there is $|t(n-1)|>t_{1-\alpha / 2}$, then $H_{0}$ is rejected, meaning that there was a significant difference between the modeling error and zero. Conversely, if there is $|t(n-1)| \leq t_{1-\alpha / 2}$, then $H_{0}$ is accepted, meaning that there was no significant difference between them. Consequently, the model is considered to pass the significance test.

From the above, the residual error, posterior variance, correlation degree and significance can be used for testing the reliability and accuracy of prediction models. Four accuracy test indices for each model are comprehensively evaluated in Table 1.

TABLE 1 The commonly used scales of different test indices for accuracy test [9,20]

\begin{tabular}{lcccc}
\hline \multirow{2}{*}{ Accuracy class } & \multicolumn{4}{c}{ Accuracy test index } \\
\cline { 2 - 5 } & $\begin{array}{c}\text { Average relative error } \\
\varepsilon(\text { avg) }\end{array}$ & $\begin{array}{c}\text { Posterior variance ratio } \\
\text { 1st level (superior) }\end{array}$ & $\begin{array}{c}\text { Small error probability } \\
p\end{array}$ & $\begin{array}{c}\text { Correlation degree } \\
\xi\end{array}$ \\
\hline 2nd level (qualified) & $\leq 1 \%$ & $\leq 0.35$ & $\geq 95 \%$ & $\geq 0.90$ \\
3rd level (marginal) & $5 \% \sim 10 \%$ & $0.35 \sim 0.50$ & $80 \% \sim 95 \%$ & $0.80 \sim 0.90$ \\
4th level (disqualified) & $10 \% \sim 20 \%$ & $0.50 \sim 0.65$ & $70 \% \sim 80 \%$ & $0.70 \sim 0.80$ \\
\hline
\end{tabular}

\section{Empirical analysis on plasma concentration of acetylsalicylic acid}

\subsection{Data}

Plasma concentration data of acetylsalicylic acid were obtained from another study [38]. This study examined four experimental rabbits whose weights were between $2.5 \mathrm{~kg}$ and $3.0 \mathrm{~kg}$. After a 12 hour fast, they were given acetylsalicylic acid tablets $\left(50-100 \mathrm{mg} \cdot \mathrm{kg}^{-1}\right)$ orally. Then, the plasma concentration data at different time points after taking the medicine were obtained. Table 2 lists the acetylsalicylic acid plasma concentrations corresponding to those four experimental rabbits.

TABLE 2 Plasma concentration of acetylsalicylic acid of four groups [38] (unit: $\mu \mathrm{g} \cdot \mathrm{mL}^{-1}$ )

\begin{tabular}{ccccc}
\hline Time-point & Group 1 & Group 2 & Group 3 & Group 4 \\
\hline $1(0.15 \mathrm{~h})$ & 151.34 & 147.34 & 153.85 & 150.83 \\
\hline $2(0.25 \mathrm{~h})$ & 169.29 & 157.94 & 170.34 & 167.34 \\
\hline $3(0.50 \mathrm{~h})$ & 211.14 & 205.78 & 211.67 & 198.23 \\
\hline $4(1.00 \mathrm{~h})$ & 232.52 & 221.45 & 236.47 & 228.46 \\
\hline $5(1.50 \mathrm{~h})$ & 247.01 & 250.63 & 258.65 & 249.85 \\
\hline$(2.00 \mathrm{~h})$ & 252.33 & 259.85 & 266.24 & 261.85 \\
$7(2.50 \mathrm{~h})$ & 255.87 & 264.46 & 273.45 & 270.46 \\
\hline $8(3.00 \mathrm{~h})$ & 258.42 & 269.74 & 277.46 & 275.27 \\
$9(3.50 \mathrm{~h})$ & 231.21 & 249.83 & 257.53 & 252.99 \\
$10(4.00 \mathrm{~h})$ & 211.85 & 228.73 & 237.35 & 230.53 \\
\hline
\end{tabular}




\begin{tabular}{lcccc}
$11(6.00 \mathrm{~h})$ & 170.36 & 192.35 & 209.35 & 201.75 \\
\hline $12(13.0 \mathrm{~h})$ & 126.55 & 143.63 & 151.43 & 149.64 \\
$13(22.0 \mathrm{~h})$ & 93.43 & 106.42 & 111.34 & 109.25 \\
\hline
\end{tabular}

Testing the significance of differences between the four groups of plasma time-concentration data should be carried out before conducting prediction. Above all, Friedman rank sum testing was conducted for the four groups of data as a whole. The Friedman chi-squared was 47.473, and the p-value was far less than 0.00001. Results indicate that there existed significant differences and high correlations among the four experimental groups. Wilcoxon signed-rank testing was then employed on each data pair. Testing results showed that there existed significant differences between each two experimental groups, except between group 1 and group 2. Therefore, the four groups of plasma time-concentration data have passed the significance testing.

Figure 2 shows the four groups of plasma concentration trend curves of acetylsalicylic acid. As is seen in this figure, there was an obvious single-peak tendency for each group of plasma concentrations, accompanied by irregular fluctuations due to the unstable changes occurring from the experimental and material factors. In conclusion, the plasma concentrations of acetylsalicylic acid showed an obvious nonlinear characteristic of single-peak tendency with stochastic fluctuations.
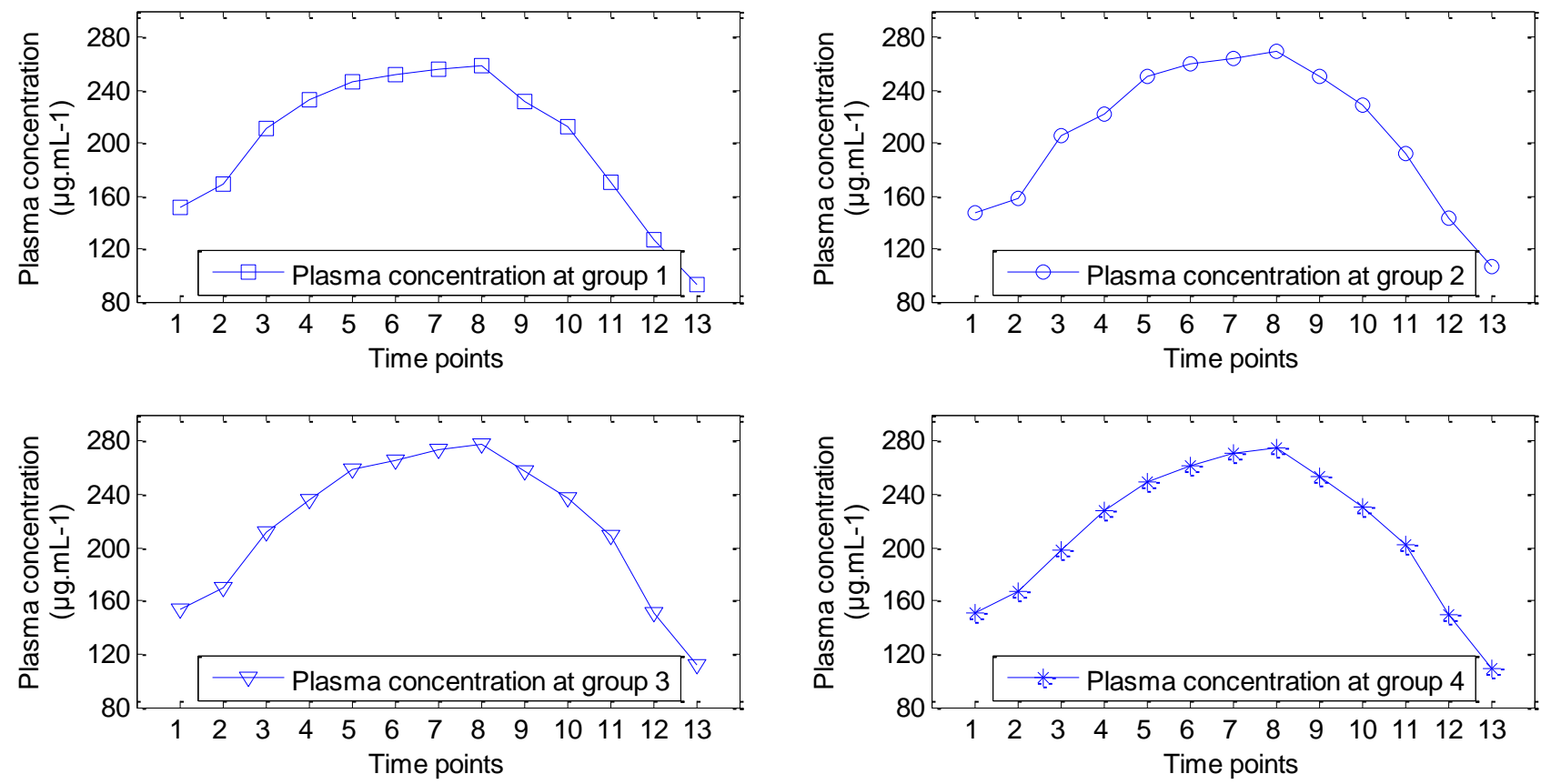

FIGURE 2 Plasma concentration tendency of acetylsalicylic acid of four groups.

\subsection{Results}

As a result of factors introducing uncertainty, such as the precision of detection and experimental error, the plasma concentrations of acetylsalicylic acid present typical grey system characteristics with small sample sizes and poor information. For this reason, this paper will build the SA-NGBM, an optimized NGBM (ONGBM) [36], an exponential smoothing model (ES) [39] and a simple moving average model (SMA) [40] to simulate the plasma concentration of acetylsalicylic acid at different time points. The plasma concentration data of the first eleven time points are taken as the simulation samples, and the data of the latter two time points are chosen as testing samples for prediction testing. Meanwhile, four popular accuracy and significance test criteria are adopted to check the prediction accuracy of different prediction models.

3.2.1. Forecasting the plasma concentration of acetylsalicylic acid of group 1

Following the modeling steps to couple the SA-NGBM with the SA algorithm, the power exponent is $\gamma=0.573411$, the interpolated coefficient of background value is $\phi=0.594408$, and the corresponding values 
of the parameter $a, b$ are $0.313723,10.804450$, respectively. Meanwhile, the value of the retrospective order is determined as $p=2$ by trial calculation method by minimizing the root-mean-square error. The memory coefficients are $\alpha_{-3}=0.403296, \alpha_{-2}=-1.846963, \alpha_{-1}=2.395267, \theta_{-2}=0.414322, \theta_{-1}=0.251846$ and $\theta_{0}=0.040747$, respectively. The model values of the SA-NGBM and other three compared models are shown in Table 3. There are no simulated values of the first two time points owing to the retrospective order $p=2$ in the SA-NGBM.

TABLE 3 The predicted values for plasma concentration of acetylsalicylic acid of group 1 (unit: $\mu \mathrm{g} \cdot \mathrm{mL}^{-1}$ )

\begin{tabular}{|c|c|c|c|c|c|c|c|c|c|}
\hline \multirow[t]{2}{*}{$\begin{array}{l}\text { Time- } \\
\text { point }\end{array}$} & \multirow{2}{*}{$\begin{array}{c}\text { Plasma } \\
\text { concentration } \\
\text { at group } 1 \\
f_{t}^{0}\end{array}$} & \multicolumn{2}{|c|}{$\begin{array}{l}\text { SA-NGBM model } \\
\gamma=0.573411 \\
\phi=0.594408, p=2\end{array}$} & \multicolumn{2}{|c|}{$\begin{array}{l}\text { ONGBM model } \\
\gamma=0.595842 \\
\phi=0.5\end{array}$} & \multicolumn{2}{|c|}{$\begin{array}{l}\text { Single exponential } \\
\text { smoothing }\end{array}$} & \multicolumn{2}{|c|}{ Simple moving average } \\
\hline & & $\hat{f}_{t}^{0}$ & $\varepsilon(t)$ & $\hat{f}_{t}^{0}$ & $\varepsilon(t)$ & $\hat{f}_{t}^{0}$ & $\varepsilon(t)$ & $\hat{f}_{t}^{0}$ & $\varepsilon(t)$ \\
\hline 2 & 169.29 & - & - & 169.290 & $0.000 \%$ & 169.290 & $0.000 \%$ & - & - \\
\hline 3 & 211.14 & - & - & 212.093 & $-0.451 \%$ & 169.290 & $19.821 \%$ & - & - \\
\hline 4 & 232.52 & 232.593 & $-0.031 \%$ & 238.766 & $-2.686 \%$ & 206.955 & $10.995 \%$ & - & - \\
\hline 5 & 247.01 & 244.099 & $1.178 \%$ & 251.908 & $-1.983 \%$ & 229.964 & $6.901 \%$ & 204.317 & $17.284 \%$ \\
\hline 6 & 252.33 & 259.987 & $-3.035 \%$ & 254.530 & $-0.872 \%$ & 245.305 & $2.784 \%$ & 230.223 & $8.761 \%$ \\
\hline 7 & 255.87 & 254.026 & $0.721 \%$ & 249.426 & $2.518 \%$ & 251.628 & $1.658 \%$ & 243.953 & $4.657 \%$ \\
\hline 8 & 258.42 & 246.525 & $4.603 \%$ & 238.955 & $7.532 \%$ & 255.446 & $1.151 \%$ & 251.737 & $2.586 \%$ \\
\hline 9 & 231.21 & 244.214 & $-5.624 \%$ & 225.007 & $2.683 \%$ & 258.123 & $11.640 \%$ & 255.540 & $-10.523 \%$ \\
\hline 10 & 211.85 & 206.735 & $2.414 \%$ & 209.035 & $1.329 \%$ & 233.901 & $10.409 \%$ & 248.500 & $-17.300 \%$ \\
\hline \multirow[t]{2}{*}{11} & 170.36 & 172.230 & $-1.098 \%$ & 192.124 & $-12.775 \%$ & 214.055 & $25.649 \%$ & 233.827 & $-37.255 \%$ \\
\hline & $\varepsilon(a v g)$ & & $2.338 \%$ & & $3.283 \%$ & & $9.101 \%$ & & $14.052 \%$ \\
\hline 12 & 126.55 & 137.129 & $-8.360 \%$ & 175.058 & $-38.331 \%$ & 174.730 & $-38.072 \%$ & 204.473 & $-61.575 \%$ \\
\hline 13 & 93.43 & 79.610 & $14.792 \%$ & 128.968 & $-38.037 \%$ & 131.368 & $-40.606 \%$ & 169.587 & $-81.512 \%$ \\
\hline
\end{tabular}

The accuracy and significance test criteria values of different prediction models for plasma concentration of acetylsalicylic acid of group 1 are listed in Table 4.

TABLE 4 Accuracy and significance test of predicted values for different grey prediction models

\begin{tabular}{lcccccc}
\hline \multicolumn{1}{c}{ Model } & RMSE & $\varepsilon($ avg $)$ & $C$ & $p$ & $\xi$ & Significance $(\alpha=0.95)$ \\
\hline SA-NGBM model & 7.165581 & $2.338 \%$ & 0.259230 & $100.000 \%$ & 0.9896 & significant \\
ONGBM model & 10.046072 & $3.283 \%$ & 0.320229 & $90.000 \%$ & 0.9911 & significant \\
Single exponential smoothing & 24.272530 & $9.101 \%$ & 0.773521 & $50.000 \%$ & 0.9745 & nonsignificant \\
Simple moving average & 34.767227 & $14.052 \%$ & 1.159815 & $42.857 \%$ & 0.6435 & nonsignificant \\
\hline
\end{tabular}

\subsubsection{Forecasting the plasma concentration of acetylsalicylic acid of group 2}

In the same way as group 1 , the power exponent $\gamma=0.631898$, background value $\phi=0.527600$, and parameter $a=0.365241, b=7.925877$ are obtained for group 2. Meanwhile, under the retrospective order $p=2$, the corresponding memory coefficients are $\alpha_{-3}=0.109115, \alpha_{-2}=-1.580079, \alpha_{-1}=2.419673$, $\theta_{-2}=-0.066091, \theta_{-1}=0.302783$ and $\theta_{0}=0.177644$, respectively. The model values of the SA-NGBM and other three compared models are shown in Table 5. Similarly, there are no simulated values of the first two time points in the SA-NGBM.

TABLE 5 The predicted values for plasma concentration of acetylsalicylic acid of group 2 (unit: $\mu \mathrm{g} \cdot \mathrm{mL}^{-1}$ )

\begin{tabular}{ccllll}
\hline Time- & Plasma & SA-NGBM model & ONGBM model & Single exponential & Simple moving average \\
point & concentration & $\gamma=0.631898$ & $\gamma=0.639316$ & smoothing & \\
& at group 2 & $\phi=0.527600, p=2$ & $\phi=0.5$ & & \\
\cline { 3 - 5 } & & &
\end{tabular}




\begin{tabular}{cccccccccc} 
& $f_{t}^{0}$ & $\hat{f}_{t}^{0}$ & $\varepsilon(t)$ & $\hat{f}_{t}^{0}$ & $\varepsilon(t)$ & $\hat{f}_{t}^{0}$ & $\varepsilon(t)$ & $\hat{f}_{t}^{0}$ & $\varepsilon(t)$ \\
\hline 2 & 157.94 & - & - & 157.940 & $0.000 \%$ & 157.940 & $0.000 \%$ & - & - \\
3 & 205.78 & - & - & 202.751 & $1.472 \%$ & 157.940 & $23.248 \%$ & - & - \\
4 & 221.45 & 221.054 & $0.179 \%$ & 234.062 & $-5.695 \%$ & 200.996 & $9.236 \%$ & - & - \\
5 & 250.63 & 250.475 & $0.062 \%$ & 252.688 & $-0.821 \%$ & 219.405 & $12.459 \%$ & 195.057 & $22.173 \%$ \\
\hline 6 & 259.85 & 260.314 & $-0.179 \%$ & 260.579 & $-0.281 \%$ & 247.507 & $4.750 \%$ & 225.953 & $13.045 \%$ \\
7 & 264.46 & 271.012 & $-2.478 \%$ & 259.983 & $1.693 \%$ & 258.616 & $2.210 \%$ & 243.977 & $7.745 \%$ \\
8 & 269.74 & 256.322 & $4.974 \%$ & 253.049 & $6.188 \%$ & 263.876 & $2.174 \%$ & 258.313 & $4.236 \%$ \\
\hline 9 & 249.83 & 256.739 & $-2.765 \%$ & 241.646 & $3.276 \%$ & 269.154 & $-7.735 \%$ & 264.683 & $-5.945 \%$ \\
10 & 228.73 & 229.996 & $-0.553 \%$ & 227.315 & $0.619 \%$ & 251.762 & $-10.070 \%$ & 261.343 & $-14.258 \%$ \\
11 & 192.35 & 191.453 & $0.466 \%$ & 211.275 & $-9.839 \%$ & 231.033 & $-20.111 \%$ & 249.433 & $-29.677 \%$ \\
\hline 12 & $\varepsilon($ avg & & & $1.457 \%$ & & $2.988 \%$ & & $9.199 \%$ & $13.869 \%$ \\
\hline 13 & 143.63 & 153.590 & $-6.935 \%$ & 194.456 & $-35.387 \%$ & 196.218 & $-36.614 \%$ & 223.637 & $-55.704 \%$ \\
\hline
\end{tabular}

The accuracy and significance test criteria values of different prediction models for plasma concentration of acetylsalicylic acid of group 2 are listed in Table 6.

TABLE 6 Accuracy and significance test of predicted values for different grey prediction models

\begin{tabular}{lcccccc}
\hline \multicolumn{1}{c}{ Model } & RMSE & $\varepsilon(a v g)$ & $C$ & $p$ & $\xi$ & Significance $(\alpha=0.95)$ \\
\hline SA-NGBM model & 5.847106 & $1.457 \%$ & 0.238907 & $100.000 \%$ & 0.9898 & significant \\
ONGBM model & 9.480363 & $2.988 \%$ & 0.276589 & $100.000 \%$ & 0.9937 & significant \\
Single exponential smoothing & 25.106145 & $9.199 \%$ & 0.721896 & $50.000 \%$ & 0.9561 & nonsignificant \\
Simple moving average & 36.508674 & $13.869 \%$ & 1.469318 & $14.286 \%$ & 0.5166 & nonsignificant \\
\hline
\end{tabular}

3.2.3. Forecasting the plasma concentration of acetylsalicylic acid of group 3

In the same way as groups 1 and 2 , the power exponent $\gamma=0.582311$, background value $\phi=0.550247$, and parameter $a=0.290872, b=9.948943$ are obtained. Meanwhile, under the retrospective order $p=2$, the corresponding memory coefficients are $\alpha_{-3}=0.341151, \alpha_{-2}=-0.934582, \alpha_{-1}=1.570336$, $\theta_{-2}=-7.756000, \theta_{-1}=18.737145$ and $\theta_{0}=-9.831399$, respectively. The model values of the SA-NGBM and other three compared models are shown in Table 7. Similarly, there are no simulated values of the first two time points in the SA-NGBM.

TABLE 7 The predicted values for plasma concentration of acetylsalicylic acid of group 3 (unit: $\mu \mathrm{g} \cdot \mathrm{mL}^{-1}$ )

\begin{tabular}{|c|c|c|c|c|c|c|c|c|c|}
\hline \multirow[t]{2}{*}{$\begin{array}{l}\text { Time- } \\
\text { point }\end{array}$} & \multirow{2}{*}{$\begin{array}{c}\text { Plasma } \\
\text { concentration } \\
\text { at group } 3 \\
f_{t}^{0}\end{array}$} & \multicolumn{2}{|c|}{$\begin{array}{l}\text { SA-NGBM model } \\
\gamma=0.582311 \\
\phi=0.550247, p=2\end{array}$} & \multicolumn{2}{|c|}{$\begin{array}{l}\text { ONGBM model } \\
\gamma=0.607507 \\
\phi=0.5\end{array}$} & \multicolumn{2}{|c|}{$\begin{array}{l}\text { Single exponential } \\
\text { smoothing }\end{array}$} & \multicolumn{2}{|c|}{ Simple moving average } \\
\hline & & $\hat{f}_{t}^{0}$ & $\varepsilon(t)$ & $\hat{f}_{t}^{0}$ & $\varepsilon(t)$ & $\hat{f}_{t}^{0}$ & $\varepsilon(t)$ & $\hat{f}_{t}^{0}$ & $\varepsilon(t)$ \\
\hline 2 & 170.34 & - & - & 166.534 & $2.234 \%$ & 170.340 & $0.000 \%$ & - & - \\
\hline 3 & 211.67 & - & - & 211.670 & $0.000 \%$ & 170.340 & $19.526 \%$ & - & - \\
\hline 4 & 236.47 & 236.358 & $0.047 \%$ & 242.731 & $-2.648 \%$ & 207.537 & $12.235 \%$ & - & - \\
\hline 5 & 258.65 & 257.480 & $0.452 \%$ & 261.155 & $-0.968 \%$ & 233.577 & $9.694 \%$ & 206.160 & $20.294 \%$ \\
\hline 6 & 266.24 & 269.010 & $-1.040 \%$ & 269.123 & $-1.083 \%$ & 256.143 & $3.792 \%$ & 235.597 & $11.510 \%$ \\
\hline 7 & 273.45 & 276.333 & $-1.054 \%$ & 268.902 & $1.663 \%$ & 265.230 & $3.006 \%$ & 253.787 & $7.191 \%$ \\
\hline 8 & 277.46 & 266.057 & $4.110 \%$ & 262.561 & $5.370 \%$ & 272.628 & $1.742 \%$ & 266.113 & $4.090 \%$ \\
\hline 9 & 257.53 & 267.177 & $-3.746 \%$ & 251.864 & $2.200 \%$ & 276.977 & $-7.551 \%$ & 272.383 & $-5.767 \%$ \\
\hline 10 & 237.35 & 233.946 & $1.434 \%$ & 238.254 & $-0.381 \%$ & 259.475 & $-9.322 \%$ & 269.480 & $-13.537 \%$ \\
\hline
\end{tabular}




\begin{tabular}{rrrrrrrrrr}
11 & 209.35 & 210.872 & $-0.727 \%$ & 222.870 & $-6.458 \%$ & 239.562 & $-14.431 \%$ & 257.447 & $-22.974 \%$ \\
\hline 12 & $\varepsilon($ avg $)$ & & $1.576 \%$ & & $2.301 \%$ & & $8.130 \%$ & & $12.195 \%$ \\
\hline 13 & 151.43 & 183.598 & $-21.243 \%$ & 206.587 & $-36.424 \%$ & 212.371 & $-40.244 \%$ & 234.743 & $-55.017 \%$ \\
\hline
\end{tabular}

The accuracy and significance test criteria values of different prediction models for plasma concentration of acetylsalicylic acid at group 3 are listed in Table 8 .

TABLE 8 Accuracy and significance test of predicted values for different grey prediction models

\begin{tabular}{lcccccc}
\hline \multicolumn{1}{c}{ Model } & RMSE & $\varepsilon(a v g)$ & $C$ & $p$ & $\xi$ & Significance $(\alpha=0.95)$ \\
\hline SA-NGBM model & 5.638736 & $1.576 \%$ & 0.263565 & $100.000 \%$ & 0.9971 & significant \\
ONGBM model & 7.257053 & $2.301 \%$ & 0.224003 & $100.000 \%$ & 0.9811 & significant \\
Single exponential smoothing & 22.687607 & $8.130 \%$ & 0.685827 & $50.000 \%$ & 0.9543 & nonsignificant \\
Simple moving average & 33.329205 & $12.195 \%$ & 1.511051 & $14.286 \%$ & 0.5109 & nonsignificant \\
\hline
\end{tabular}

\subsubsection{Forecasting the plasma concentration of acetylsalicylic acid of group 4}

In the same way as the prior groups, the power exponent $\gamma=0.665516$, background value $\phi=0.607350$, and parameter $a=0.416486, b=6.833010$ are obtained. Meanwhile, under the retrospective order $p=2$, the corresponding memory coefficients are $\alpha_{-3}=-0.082427, \alpha_{-2}=-0.518745, \alpha_{-1}=1.572655$, $\theta_{-2}=-5.859309, \theta_{-1}=13.065967$ and $\theta_{0}=-6.406394$, respectively. The model values of the SA-NGBM and other three compared models are shown in Table 9. Similarly, there are no simulated values of the first two time points in the SA-NGBM.

TABLE 9 The predicted values for plasma concentration of acetylsalicylic acid of group 4 (unit: $\mu \mathrm{g} \cdot \mathrm{mL}^{-1}$ )

\begin{tabular}{|c|c|c|c|c|c|c|c|c|c|}
\hline \multirow[t]{2}{*}{$\begin{array}{l}\text { Time- } \\
\text { point }\end{array}$} & \multirow{2}{*}{$\begin{array}{c}\text { Plasma } \\
\text { concentration } \\
\text { at group } 4 \\
f_{t}^{0}\end{array}$} & \multicolumn{2}{|c|}{$\begin{array}{l}\text { SA-NGBM model } \\
\gamma=0.665516 \\
\phi=0.607350, p=2\end{array}$} & \multicolumn{2}{|c|}{$\begin{array}{l}\text { ONGBM model } \\
\gamma=0.716306 \\
\phi=0.5\end{array}$} & \multicolumn{2}{|c|}{$\begin{array}{l}\text { Single exponential } \\
\text { smoothing }\end{array}$} & \multicolumn{2}{|c|}{ Simple moving average } \\
\hline & & $\hat{f}_{t}^{0}$ & $\varepsilon(t)$ & $\hat{f}_{t}^{0}$ & $\varepsilon(t)$ & $\hat{f}_{t}^{0}$ & $\varepsilon(t)$ & $\hat{f}_{t}^{0}$ & $\varepsilon(t)$ \\
\hline 2 & 167.34 & - & - & 151.319 & $9.574 \%$ & 167.340 & $0.000 \%$ & - & - \\
\hline 3 & 198.23 & - & - & 198.230 & $0.000 \%$ & 167.340 & $15.583 \%$ & - & - \\
\hline 4 & 228.46 & 228.366 & $0.041 \%$ & 233.284 & $-2.112 \%$ & 195.141 & $14.584 \%$ & - & - \\
\hline 5 & 249.85 & 247.001 & $1.140 \%$ & 255.542 & $-2.278 \%$ & 225.128 & $9.895 \%$ & 198.010 & $20.748 \%$ \\
\hline 6 & 261.85 & 269.918 & $-3.081 \%$ & 266.057 & $-1.607 \%$ & 247.378 & $5.527 \%$ & 225.513 & $13.877 \%$ \\
\hline 7 & 270.46 & 268.270 & $0.810 \%$ & 266.806 & $1.351 \%$ & 260.403 & $3.718 \%$ & 246.720 & $8.778 \%$ \\
\hline 8 & 275.27 & 264.234 & $4.009 \%$ & 260.027 & $5.537 \%$ & 269.454 & $2.113 \%$ & 260.720 & $5.286 \%$ \\
\hline 9 & 252.99 & 262.799 & $-3.877 \%$ & 247.852 & $2.031 \%$ & 274.688 & $-8.577 \%$ & 269.193 & $-6.405 \%$ \\
\hline 10 & 230.53 & 230.290 & $0.104 \%$ & 232.124 & $-0.691 \%$ & 255.160 & $-10.684 \%$ & 266.240 & $-15.490 \%$ \\
\hline 11 & 201.75 & 200.155 & $0.791 \%$ & 214.339 & $-6.240 \%$ & 232.993 & $-15.486 \%$ & 252.930 & $-25.368 \%$ \\
\hline & $\varepsilon(a v g)$ & & $1.732 \%$ & & $3.142 \%$ & & $8.617 \%$ & & $13.707 \%$ \\
\hline 12 & 149.64 & 169.648 & $-13.371 \%$ & 195.654 & $-30.750 \%$ & 204.874 & $-36.911 \%$ & 228.423 & $-52.648 \%$ \\
\hline 13 & 109.25 & 106.204 & $2.788 \%$ & 152.553 & $-39.637 \%$ & 155.163 & $-42.026 \%$ & 193.973 & $-77.550 \%$ \\
\hline
\end{tabular}

The accuracy and significance test criteria values of different prediction models for plasma concentration of acetylsalicylic acid at group 3 are listed in Table 10.

TABLE 10 Accuracy and significance test of predicted values for different grey prediction models

\begin{tabular}{lcccccc}
\hline \multicolumn{1}{c}{ Model } & RMSE & $\varepsilon($ avg $)$ & $C$ & $p$ & $\xi$ & Significance $(\alpha=0.95)$ \\
\hline SA-NGBM model & 6.109683 & $1.732 \%$ & 0.264338 & $100.000 \%$ & 0.9957 & significant \\
ONGBM model & 8.735930 & $3.142 \%$ & 0.258615 & $100.000 \%$ & 0.9138 & significant
\end{tabular}




\begin{tabular}{lllllll} 
Single exponential smoothing & 22.524427 & $8.617 \%$ & 0.660678 & $50.000 \%$ & 0.9557 & nonsignificant \\
Simple moving average & 35.737509 & $13.707 \%$ & 1.506252 & $14.286 \%$ & 0.5299 & nonsignificant \\
\hline
\end{tabular}

\subsection{Discussion}

From the viewpoint of accuracy and significance testing, the nonlinear grey Bernoulli model is particularly appropriate to approximate single-peak sequences. It is shown that the NGBM can take into full consideration the characteristics of approximating single-peaks among sequences and is able to adequately reflect the integral evolution laws of nonlinear systems compared with the ES [39] and SMA [40] models. Nevertheless, as a result of uncertainty about external disturbances, the traditional NGBM $[24,25]$ cannot effectively reflect large stochastic fluctuations that commonly exist in nonlinear systems. In contrast, the SA method helped the NGBM to closely catch individual stochastic variations. The limitations of the traditional NGBM, such as sensitivity to initial values of the differential equation, can be eliminated by using multi-timepoint initial fields instead using data from only a single time point [30]. The prediction accuracy of the SA-NGBM was further improved compared with the traditional ONGBM. Meanwhile, the fitting curves of plasma concentration of acetylsalicylic acid and their corresponding comparison results of the $\mathrm{RPE}_{t}$ distribution are shown in Figures 3-6 respectively. As seen from the following figures, the proposed SA-NGBM model can better represent the tendency of integral single-peak development, along with individual fluctuations existing in nonlinear systems, and possesses more stable and ideal prediction effects. Therefore, it is a reliable and stable model for predicting the future evolutionary trend of single-peaked nonlinear systems with small sample sizes.
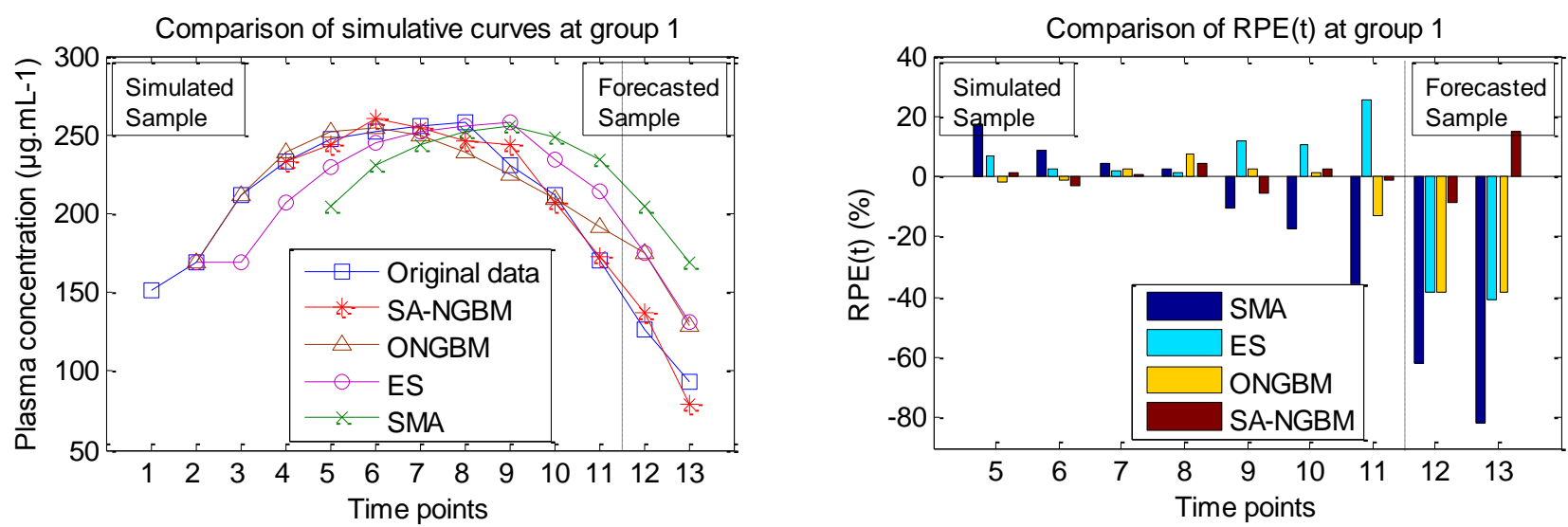

FIGURE 3 Comparison among original and simulative curves of different prediction models of group 1 .
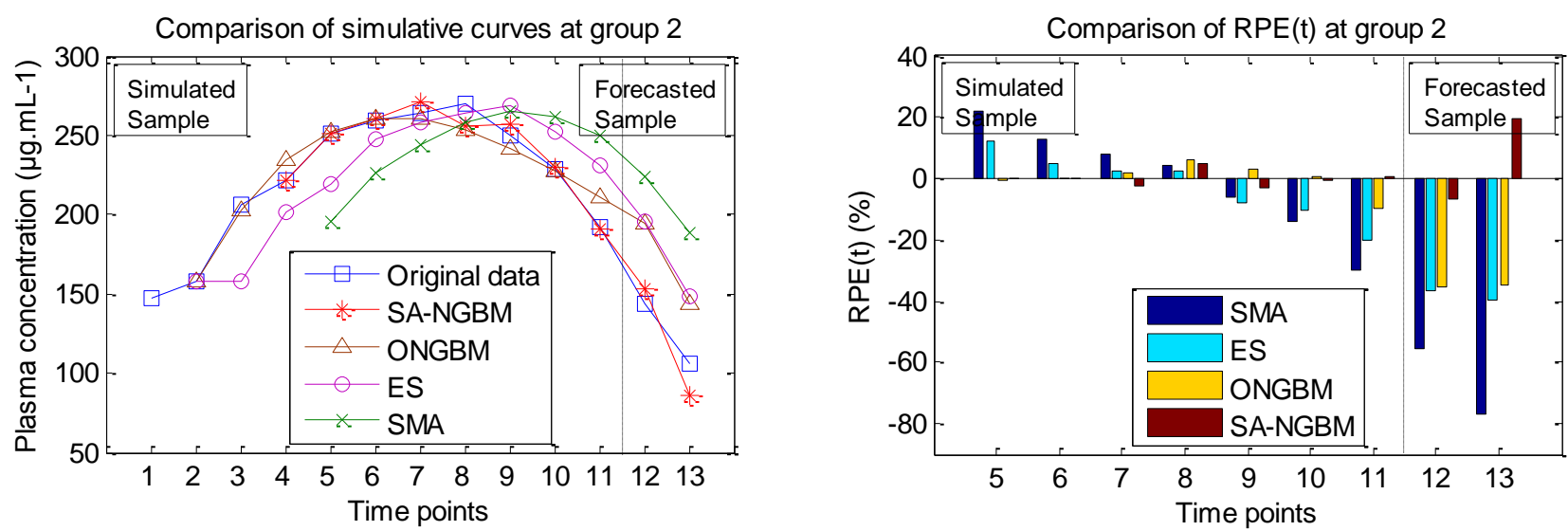

FIGURE 4 Comparison among original and simulative curves of different prediction models of group 2. 

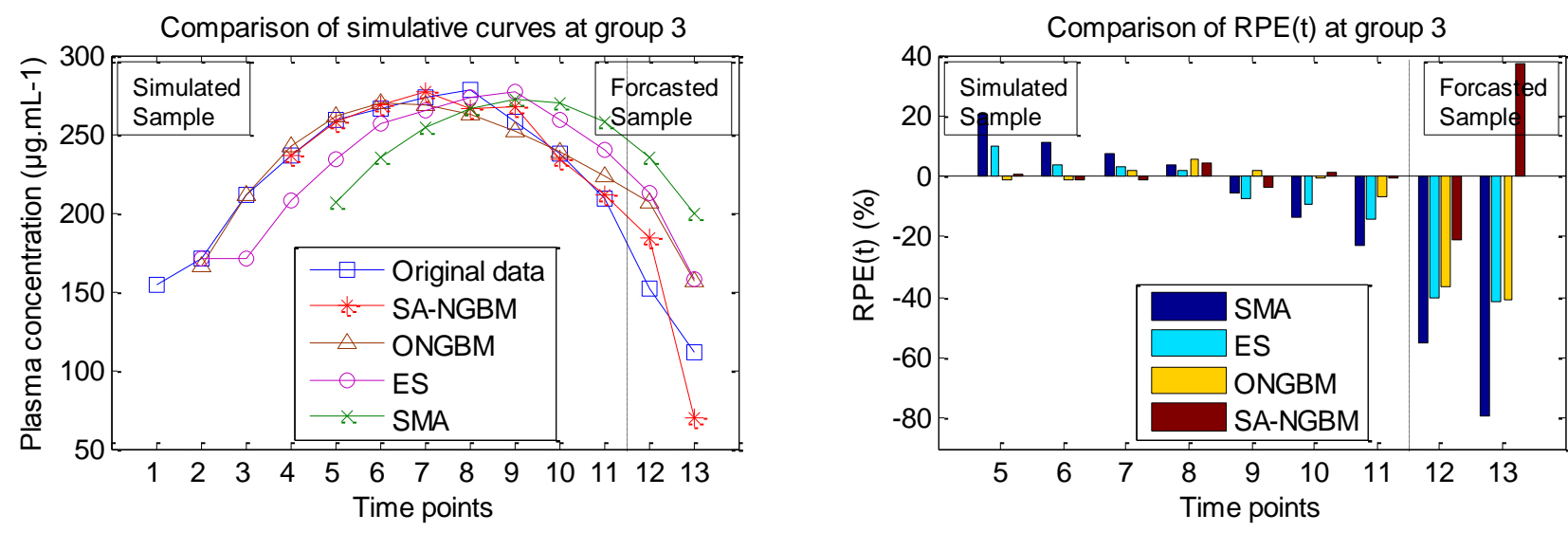

FIGURE 5 Comparison among original and simulative curves of different prediction models of group 3.
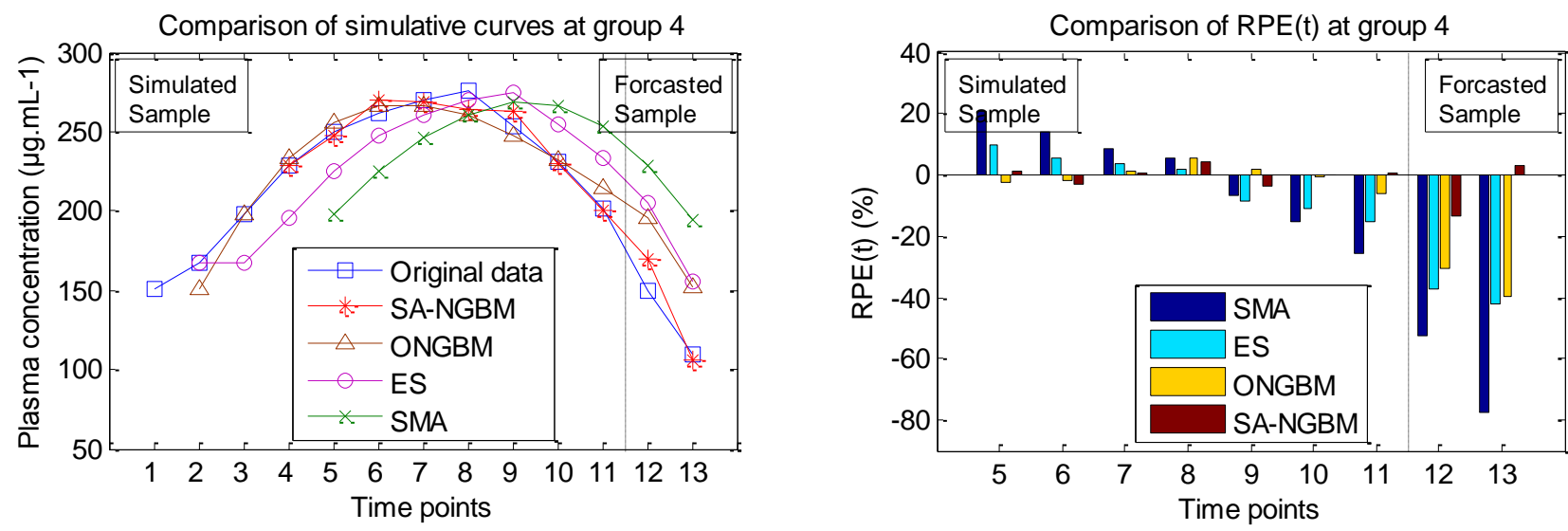

FIGURE 6 Comparison among original and simulative curves of different prediction models of group 4.

In addition, from the comparison of results across accuracy and significance tests, the residual error, posterior variance, correlation degree and error significance of the SA-NGBM were widely superior to that of other prediction models. Thus, according to grey prediction theory $[9,20]$, the proposed SA-NGBM could be used to carry out extrapolation and prediction that could reasonably reflect the decreasing trend of the future plasma concentration of acetylsalicylic acid.

Nevertheless, in real-life situations, there exist numerous influence factors involved in plasma concentration. For example, the individual difference between patients, different dosage regimens, the unknown potential drug interactions, the pathophysiological status of patients, and so forth, could all influence plasma concentrations. Therefore, high-quality plasma time-concentration data are difficult to acquire. With the development of clinical drug trials, the systematic population pharmacokinetics can be conducted in the clinical stage of new drugs. Consequently, the high-quality data samples can be obtained for conducting plasma concentration prediction and guiding clinical medication regimens.

\section{Conclusion and future work}

In this paper, we propose a self-memory algorithm-based NGBM model termed SA-NGBM and utilize it to predict the plasma concentration of acetylsalicylic acid. The model combines the prediction advantages of the self-memory algorithm and the traditional NGBM. SA could be useful for catching the individual stochastic fluctuations existing in nonlinear systems due to uncertain external disturbances. The system's self-memorization equation of the SA-NGBM contains a multiple-time-point initial field instead of an initial value determined from a single time point. Consequently, it overcomes the limitations of being sensitive to the initial value used in the traditional NGBM and possesses more robust predictions. The empirical results indicated that the new model can take full advantage of the historical statistical information and capture tightly the nonlinear system's tendency of 
integral evolution and individual fluctuations. It is worth popularizing and applying to forecast other single-peaked time sequences with similar sample sizes.

Plasma concentration prediction and monitoring for drugs are important to guide the rational use of drugs as clinical medications. Accurate predictions are conducive to reduce the adverse reactions of patients, and are expected to provide more convenience for drug developers. In clinical environments, the original plasma time-concentration data distributions often present downward trends, such as intravenous administration, or the single-peaked types, such as extravascular administration. Nevertheless, many plasma time-concentration data that are required by conventional pharmacokinetics cannot be acquired easily. Under the condition of small sample sizes, the clinical plasma concentration sequence characteristics of single-peaked series can also be predicted using analogical modeling. Once the accuracy and significance test has been passed, the model can be used for extrapolation forecasting. Accordingly, through the prediction method proposed in this paper, similar small sample-size, single-peaked, plasma concentration series of other drugs can be predicted by analogy. Patients can be individually tracked and treated, allowing for drug dosage, physical metabolism and other drug guidance programs to be obtained more scientifically and accurately. By means of the combination of computer algorithms and clinical pharmacology, data-mining methods can be used to improve human health and have good development prospects.

However, the existing research on the SA-NGBM is not comprehensive in this paper. Problems such as the intelligent optimization algorithms of the retrospective order have not been discussed. Additionally, the SA-NGBM is a univariable nonlinear grey Bernoulli model and cannot reflect the effect of relevant factors in multivariable nonlinear systems with interacting relationships. In addition, new plasma time-concentration data will continually enter into the original systems as a result of the periodical monitoring when considering the time-concentration curve for repeated administrations. The metabolism model based on the new information priority principle may be introduced into the SA-NGBM. Therefore, there remain some solutions that should be explored for these problems.

\section{Acknowledgments}

The authors are grateful to the editors and the anonymous referees for their constructive comments and suggestions that helped to improve the clarity and completeness of this paper. This work was supported by a Marie Curie International Incoming Fellowship within the 7th European Community Framework Programme (No. FP7-PIIF-GA-2013-629051), the National Natural Science Foundation of China (Nos. 71603135 and 71871084), the Humanistic and Social Science Youth Foundation of Ministry of Education of China (Nos. 15YJC630048 and 18YJC630043), the Funding of Nantong Science and Technology Program (No. MS12015013) and the Jiangsu Government Scholarship for Overseas Studies.

\section{References}

[1] J.H. Yang, Physiological-atrium model, J. Biomath. 14 (4) (1999) 440-444.

[2] P. Neelamegam, A. Jamaludeen, A. Rajendran, Prediction of calcium concentration in human blood serum using an artificial neural network, Measurement 44 (2011) 312-319.

[3] A. Wiesel, O. Bibi, A. Globerson, Time varying autoregressive moving average models for covariane estimation, IEEE Trans. Signal Process. 61 (2013) 2791-2801.

[4] T. Matsubara, J. Morimoto, Bilinear modeling of EMG signals to extract user-independent features for multiuser myoelectric interface, IEEE Trans. Biomed. Engin. 60 (2013) 2205-2213.

[5] G. Li, C. Wen, W. Zheng, Y. Chen, Identification of a class of nonlinear autoregressive models with exogenous inputs based on kernel machines, IEEE Trans. Signal Process. 59 (5) (2011) 2146-2159.

[6] L.A. Zadeh, Fuzzy sets, Inform. Control 8 (1965) 338-353.

[7] Z. Pawlak, Rough sets, Int. J. Inform. Comp. Sci. 11 (1982) 341-356.

[8] J.L. Deng, Control problems of grey systems, Syst. Control Lett. 1 (5) (1982) 288-294.

[9] S.F. Liu, Grey System Theory and Its Application (8th ed), Beijing: Science Press, 2016.

[10] N.M. Xie, S.F. Liu, Y.J. Yang, C.Q. Yuan, On novel grey forecasting model based on non-homogeneous 
index sequence, Appl. Math. Model. 37 (7) (2013) 5059-5068.

[11] L. Wu, S. Liu, Y. Yang, Grey double exponential smoothing model and its application on pig price forecasting in China, Appl. Soft Comput. 39 (2016) 117-123.

[12] J. Ye, Y. Dang, B. Li, Grey-Markov prediction model based on background value optimization and central-point triangular whitenization weight function, Commun. Nonlinear Sci. Numer. Simul. 54 (2018) 320-330.

[13] S. Ding, K. Hipel, Y.G. Dang, Forecasting China's electricity consumption using a new grey prediction model, Eng. 149 (2018) 314-328.

[14] B. Zeng, H.M. Duan, Y. Bai, W. Meng, Forecasting the output of shale gas in China using an unbiased grey model and weakening buffer operator, Eng. 151 (2018) 238-249.

[15] L.F. Wu, S.F. Liu, Y.J. Yang, L.H. Ma, H.X. Liu, Multi-variable weakening buffer operator and its application, Inform. Sci. 339 (2016) 98-107.

[16] H. Guo, X.P. Xiao, J. Forrest, A research on a comprehensive adaptive grey prediction model CAGM(1,N), Appl. Math. Comput. 225 (1) (2013) 216-227.

[17] Y.T. Lee, Structure activity relationship analysis of phenolic acid phenethyl esters on oral and human breast cancers: The grey $\mathrm{GM}(0, \mathrm{~N})$ approach, Comput. Biol. Med. 41 (2011) 506-511.

[18] L.P. Zhang, Y.L. Zheng, K. Wang, X.L. Zhang, Y.J. Zheng, An optimized Nash nonlinear grey Bernoulli model based on particle swarm optimization and its application in prediction for the incidence of Hepatitis B in Xinjiang, China, Comput. Biol. Med. 49 (2014) 67-73.

[19] X.J. Shen, L.M. Ou, X.J. Chen, X. Zhang, X.R. Tan, The application of the grey disaster model to forecast epidemic peaks of typhoid and paratyphoid fever in China, PLoS One 8 (4) (2013) e60601.

[20] S.F. Liu, Y.J. Yang, J. Forrest, Grey data analysis: methods, Models and Applications, New York: Springer, 2017.

[21] N.M. Xie, S.F. Liu, Discrete grey forecasting model and its optimization, Appl. Math. Model. 33 (2) (2009) 1173-1186.

[22] L.F. Wu, S.F. Liu, L.G. Yao, S.L. Yan, D.L. Liu, Grey system model with the fractional order accumulation, Commun. Nonlinear Sci. Numer. Simul. 18 (7) (2013) 1775-1785.

[23] L.F. Wu, S.F. Liu, Z.G. Fang, H.Y. Xu, Properties of the GM(1,1) with fractional order accumulation, Appl. Math. Comput. 252 (2015) 287-293.

[24] C.I. Chen. Application of the novel nonlinear grey Bernoulli model for forecasting unemployment rate, Chaos Soliton. Fract. 37 (1) (2008) 278-287.

[25] C.I. Chen, H.L. Chen, S.P. Chen, Forecasting of foreign exchange rates of Taiwan's major trading partners by novel nonlinear Grey Bernoulli model NGBM(1,1), Commun. Nonlinear Sci. Numer. Simul. 13 (6) (2008) 1194-1204.

[26] H.X. Cao, Self-memorization equation in atmospheric motion, Sci. Chin. Ser. B 36 (7) (1993) 845-855.

[27] N. Phienwej, S. Thepparak, P.H. Giao, Prediction of differential settlement of buildings induced by land subsidence from deep well pumping, Geotech. Engin. 36 (1) (2005) 69-76.

[28] X.P. Liu, X.B. Xie, Y.Z. Luo, Self-memorization model of dynamic system for predicting nonlinear displacement of slopes, Chin. J. Geotech. Engin. 32 (2010) 1535-1542.

[29] W. Wang, J.Y. Su, B.W. Hou, J. Tian, D.H. Ma, Dynamic prediction of building subsidence deformation with data-based mechanistic self-memory model, Chin. Sci. Bull. 57 (23) (2012) 2171-2176.

[30] X.H. Fan, Y. Zhang, A novel self-memory grey model, Syst. Eng. Theory Pract. 23 (2003) 114-117.

[31] X.D. Chen, J. Xia, Q. Xu, Differential hydrological grey model (DHGM) with self-memory function and its application to flood forecasting, Sci. Chin. Ser. E: Tech. Sci. 39 (2) (2009) 341-350.

[32] X.J. Guo, S.F. Liu, Z.G. Fang, Self-memory prediction model of interval grey number based on grey degree of compound grey number, Syst. Eng. Electron. 36 (6) (2014) 1124-1129.

[33] D.G. Zill, M.R. Cullen, Advanced engineering mathematics, 3rd Edition, Massachusetts: Jones and Bartlett, 2006.

[34] Z.X. Wang, Y.G. Dang, S.F. Liu, Z.W. Lian, Solution of GM(1,1) power model and its properties, Syst. Eng. Electron. 31 (10) (2009) 2380-2383.

[35] Z.X. Wang, K.W. Hipel, Q. Wang, S.W. He, An optimized $\operatorname{NGBM}(1,1)$ model for forecasting the qualified discharge rate of industrial wastewater in China, Appl. Math. Model. 35 (12) (2011) 5524-5532.

[36] Z.X. Wang, Y.G. Dang, J.J. Zhao, Optimized GM(1,1) power model and its application, Syst. Eng. Theory 
Pract. 32 (9) (2012) 1973-1978.

[37] Z.X. Wang, An optimized Nash nonlinear grey Bernoulli model for forecasting the main economic indices of high technology enterprises in China, Comput. Ind. Eng. 64 (3) (2013) 780-787.

[38] H.H. Yan, Z.H. Zhu, L.M. Liu, Q.X. Fang, G. Wang, H.J. Zhao, Prediction of lasma concentration based on wavelet neural network time series model, Chin. J. Mod. Appl. Pharm. 33 (11) (2016) 1417-1422.

[39] B. Billah, M.L. King, R.D. Snyder, A.B. Koehler, Exponential smoothing model selection for forecasting, Int. J. Forecast. 22 (2) (2006) 239-247.

[40] F.R. Johnston, J.E. Boyland, M. Meadows, E. Shale, Some properties of a simple moving average when applied to forecasting a time series, J. Oper. Res. Soc. 50 (1999) 1267-1271. 


\title{
A prediction method for plasma concentration by using a nonlinear grey Bernoulli combined model based on a self-memory algorithm
}

\author{
Xiaojun Guo ${ }^{a^{*}}$, Sifeng Liu ${ }^{b}$, Yingjie Yang ${ }^{c}$ \\ ${ }^{\text {a }}$ School of Science, Nantong University, Nantong 226019, China \\ ${ }^{\mathrm{b}}$ College of Economics and Management, Nanjing University of Aeronautics and Astronautics, Nanjing 211106, China \\ ${ }^{\mathrm{c}}$ Centre for Computational Intelligence, De Montfort University, Leicester, LE1 9BH, UK
}

\begin{abstract}
The goal of this work is to present and explore the application of a novel nonlinear grey Bernoulli combined model based on a self-memory algorithm, abbreviated as SA-NGBM, for modeling single-peaked sequences of time samples of acetylsalicylate plasma concentration following oral dosing. The self-memorization SA-NGBM routine reduces the dependence on a solitary initial value, as the initial state of the model utilizes multiple time samples. To test its forecasting performance, the SA-NGBM was used to extrapolate the plasma concentration predicted data, in comparison with the later time samples. The results were contrasted with those of the traditional optimized NGBM (ONGBM), exponential smoothing (ES) and simple moving average (SMA) using four popular accuracy and significance tests. That comparison showed that the SA-NGBM was much more accurate and efficient for matching the individual, nonlinear-system stochastic fluctuations than the existing ONGBM, ES and SMA models. The findings have potential applications for signal matching to similar small sample size, single-peaked, plasma concentration series.
\end{abstract}

\section{Keywords}

grey prediction theory; nonlinear grey Bernoulli model; self-memory algorithm; plasma concentration.

\section{Introduction}

With the rapid development of pharmaceutical science, practice indicates that the pharmacological action of many medicines is often closely related to their plasma concentration. The relationship of the therapeutic effect or toxicity of drugs follows plasma concentrations more closely than dosage. Plasma concentration refers to the total concentration in plasma after the drug is absorbed, and it usually can reflect the concentration variation of drug at the site of action. Therefore, the plasma concentration prediction for drugs is an important concern for modern medical research and has a great significance for rational drug use in patients and clinical medication guidance. The conventional prediction methods are pharmacokinetic models, constrained by kinetic parameters for individual drugs. For instance, in a compartment model, the organism could be assumed to be an abstract one-compartment, two-compartment or multicompartment dynamical model, according to different pharmic disposal speeds. Thus, the relationship between drug concentration and time in each compartment could be expressed by one differential equation, and the drug concentration could be obtained by means of solving the corresponding differential equations [1]. However, the traditional pharmacokinetic models should be performed under the hypothesis of many drug concentration data at multiple time points. Nonlinear iterative regression used to solve for the parameters easily leads to large computational demands and complicated processes. In recent years, some artificial intelligence methods, such as artificial neural networks (ANN), have been considered to forecast plasma concentrations [2]. On the premise of large sample data, these methods can perform prediction by means of training data without assuming a drug behavior model. However, it is difficult to acquire lots of high-quality clinical data due to many influencing factors and the absence of effective monitoring information. Meanwhile, the human body is an extremely complex organism that includes various random factors, so changes in plasma concentration in the body could be considered as an irregular and uncertain nonlinear system. Moreover, the grey systems theory is particularly suitable for studying those systems containing partially known information and

\footnotetext{
* Corresponding author. Tel.: +86 13862960631.
} 
partially unknown information or underdetermined problems.

The traditional statistical prediction methods, such as the autoregressive moving-average model [3], the bilinear model [4] and the nonlinear autoregressive model [5], require assumptions about the system structure. However, there often exists uncertainty arising from the limitation of cognitive level and the influence of unknown disturbance. Hence, only part of the system structure could be fully realized, or it is very difficult to obtain the available information with large sample size. In view of the increasing complexity, uncertainty and chaos of the system's structure, several theories about uncertain systems have been produced, including fuzzy mathematics proposed by Zadeh [6] and the rough sets theory by Pawlak [7]. As an emerging branch of uncertainty systems science, the grey systems theory was initially proposed by Deng to study uncertain systems with small sample size and poor information [8,9]. Grey systems contain incomplete information which parts of the system are partially known or unknown. In this uncertainty theory, valuable information can be extracted by means of the accumulated generating operation (AGO) used on a small amount of historical observation (data). As an important theoretical component, the grey prediction models can weaken the randomness of original statistical data through AGO [10]. As a benefit over traditional statistical models, grey prediction models require only a limited amount of historical observation (data) and do not require knowing their statistical distribution. So far, they have already been effectively utilized in numerous fields, such as social economy [11,12], electric engineering [13], energy environment [14,15], transportation [16], public health [17,18,19] and have demonstrated satisfactory prediction results.

Among the grey systems, the $\operatorname{GM}(1,1)$ model is the basic core model and is especially useful for approximating homogenous exponential sequence [20]. Since first proposed by Deng, a series of improved grey prediction models were continuously developed for different types of time sequences, including the discrete grey model [21] and the $\operatorname{GM}(1,1)$ model with fractional order accumulation [22,23]. However, the original data distributions obtained from pharmacokinetic studies often present downward trends, such as from intravenous administration, or the single-peak types (an increase followed by a decrease), such as from extravascular administration. The conventional grey prediction models, as mentioned above, are inappropriate for the pharmacokinetic prediction. However, the nonlinear grey Bernoulli model termed $\operatorname{NGBM}(1,1)$ is a recently developed grey prediction model proposed by Chen, which is especially suitable for a class of approximate single-peak sequences $[24,25]$. After combining the $\operatorname{GM}(1,1)$ model with the Bernoulli differential equation, the power exponent in the NGBM can flexibly determine the trend curve of real systems and effectively manifest nonlinear characteristics. Nevertheless, certain time sequences of real systems, including nonlinear systems, often show large stochastic fluctuations due to uncertain external disturbances. Additionally, there is a limitation of these models that they are sensitive to the initial value of the original dynamic differential equation. It is inevitable that the original grey models will generate apparent modeling errors, so the self-memory algorithm could be considered for including the individual fluctuations occurring in general evolution tendencies.

Based on the irreversibility of natural and social phenomena, Cao first proposed the self-memory algorithm of dynamic systems by introducing the memory concept [26]. The history information should be investigated adequately if we want to realize the current status of system and predict its future trend. The self-memory algorithm (SA) exactly emphasizes the relationship between states before and after the current system status. It regards the environmental factors as external forcing, with a view to the evolution law of system per se. By introducing the memory function which contains historical information, the differential equation that restricts the dynamic system can be transformed into an appropriate difference-integral equation termed the self-memorization equation. It is a dynamical equation, though parameter estimation of the corresponding memory coefficients is a statistical method. Therefore, SA is the statistical-dynamic method to solve problems of nonlinear dynamic systems by integrating deterministic and random theories [27]. The predictive superiority of SA lies in that it not only avoids the drawback of using only one initial value in the initial-value problems of differential equations but also absorbs the advantage of extracting information from historical data in statistics. SA is a breakthrough for numerical solution of traditional initial-value problems and statistical methods, and it has been increasingly 
applied into various fields including meteorology, hydrology, and engineering science [28,29]. Meanwhile, SA was also combined with some grey prediction models including the $\operatorname{GM}(1,1)$ model, the $\operatorname{DHGM}(2,2)$ model and interval grey number models and showed satisfactory prediction results [30-32]. Accordingly, SA can be further introduced into the NGBM to predict the single-peaked nonlinear system together with individual stochastic fluctuations.

Aiming at the single-peaked nonlinear systems with small samples that exist in pharmacokinetic studies, this paper will construct a self-memory algorithm-based nonlinear grey Bernoulli model (SA-NGBM). The traditional NGBM can describe the integral single-peaked development tendencies of nonlinear systems, and the self-memory algorithm can capture the individual stochastic variations. The proposed SA-NGBM combines the prediction advantages of the SA and NGBM models through coupling the two prediction methods. The limitations of the traditional NGBM, such as sensitivity to the initial values of the differential equation, can be eliminated by using multi-timepoint initial field instead of an initial value from a single time point. Meanwhile, the SA-NGBM will be applied to simulate the plasma concentration of acetylsalicylic acid for method validation.

The remainder of this paper is organized as follows: A brief introduction to the general nonlinear grey Bernoulli model, the self-memory algorithm and the self-memory algorithm-based nonlinear grey Bernoulli model are presented and discussed in Section 2. In Section 3, an empirical analysis on plasma concentration of acetylsalicylic acid are adopted to verify the feasibility and effectiveness of the novel SA-NGBM proposed here. Finally, some conclusions are drawn and future work proposed in Section 4.

\section{Methods}

\subsection{General nonlinear grey Bernoulli model (NGBM)}

In grey systems theory, the NGBM is a first-order single-variable grey model with an interpolated coefficient in the background value. The basic form and characteristics of general NGBM can be summarized as follows [24]:

First, assume that the original historical time sequence

$$
F_{t}^{0}=\left\{f_{t}^{0} \mid t \in 1,2, \cdots, n\right\}
$$

is a nonnegative data sequence, where the observational data $f_{t}^{0}$ corresponds to the system input at time $t$ and $n$ is the total number of time points. Set $f_{1}^{1}=f_{1}^{0}$ and construct $F_{t}^{1}$ by a one-time accumulated generation operation (1-AGO), which is given by

$$
F_{t}^{1}=\left\{f_{t}^{1} \mid t \in 1,2, \cdots, n\right\},
$$

where $f_{t}^{1}=\sum_{k=1}^{t} f_{k}^{0}, t=1,2, \cdots, n$ and $F_{t}^{1}$ is termed as the 1-AGO sequence of $F_{t}^{0}$.

Next, based on the principle of elementary differential equations, the Bernoulli equation [33] is introduced to establish the nonlinear grey Bernoulli equation for the NGBM as the following form

$$
f_{t}^{0}+a g_{t}{ }^{1}=b\left(g_{t}\right)^{\gamma}, t=2,3, \cdots, n,
$$

where $f_{t}^{0}$ is called a grey derivative, $g_{t}^{1}=\phi \cdot f_{t}^{1}+(1-\phi) \cdot f_{t-1}^{1}$ is referred to as the background value of the grey derivative $f_{t}^{0}$, and $\phi$ is called the interpolated coefficient of the background value within a closed interval $[0,1]$, which is traditionally set to 0.5 . When $\phi$ is equal to 0.5 , Eq. (3) is the general NGBM [24]. When $\phi$ is an indefinite value in the interval [0,1], Eq. (3) is the Nash nonlinear grey Bernoulli model (NNGBM) [25].

Then, at sampling time $\Delta t=1$, the parameters $a$ and $b$ in matrix $\hat{R}$ can be obtained through least-squares estimation according to Eq. (3) as follows:

$$
\hat{R}=\left(\begin{array}{l}
\hat{a} \\
\hat{b}
\end{array}\right)=\left(B^{\mathrm{T}} B\right)^{-1} B^{\mathrm{T}} F_{n},
$$


where $B=\left[\begin{array}{cc}-g_{2}^{1} & \left(g_{2}^{1}\right)^{\gamma} \\ -g_{3}^{1} & \left(g_{3}^{1}\right)^{\gamma} \\ \vdots & \vdots \\ -g_{n}^{1} & \left(g_{n}^{1}\right)^{\gamma}\end{array}\right]$ and $F_{n}=\left[\begin{array}{c}f_{2}^{0} \\ f_{3}^{0} \\ \vdots \\ f_{n}^{0}\end{array}\right]$. On the basis of the monotonically increasing sequence $F_{t}^{1}$, the whitenization differential equation of the NGBM (i.e., a first-order differential equation) can be established as follows:

$$
\frac{\mathrm{d} F_{t}^{1}}{\mathrm{~d} t}+a F_{t}^{1}=b\left(F_{t}^{1}\right)^{\gamma}
$$

where the parameters $a$ and $b$ are called the developing and grey input coefficients, respectively, and the adjustable parameter $\gamma \neq 1$ is called the power exponent.

Thus, Eq. (5) can be solved together with initial value $\hat{f}_{1}^{1}=f_{1}^{0}$, and the corresponding particular solution is

$$
\hat{f}_{t+1}^{1}=\left[\left(\left(f_{1}^{0}\right)^{1-\gamma}-\frac{b}{a}\right) e^{-a(1-\gamma) t}+\frac{b}{a}\right]^{1 /(1-\gamma)}, \gamma \neq 1, \forall t \geq 1,
$$

which is called the time-response sequence of the NGBM. Then, the system output $\hat{f}_{t+1}^{1}$ of dependent variable $F_{t}^{1}$ can be obtained from Eq. (6), accordingly. Application of the first-order inverse accumulated generation operation (1-IAGO) to $\hat{f}_{t+1}^{1}$ allows the simulation and forecasting values of the original sequence $F_{t}^{0}$ to be obtained as follows:

$$
\hat{f}_{t+1}^{0}=\hat{f}_{t+1}^{1}-\hat{f}_{t}, \quad \forall t \geq 1 .
$$

Remark. The NGBM is a sort of significant grey prediction model especially useful for the nonlinear small-sample time sequences, where the adjustable power exponent $\gamma$ plays an important role. The adjustable value of $\gamma$ that matches with actual data could be acquired by certain optimization techniques. When $\gamma=0$, the NGBM will degenerate into the traditional $\operatorname{GM}(1,1)$ model; when $\gamma=2$, the NGBM will degenerate into the grey Verhulst model. Comparatively, the adjustable power exponent $\gamma$ in the NGBM can flexibly fit the actual historical observation (data) and effectively reflect the nonlinear characteristics of real systems. Numerous correlational studies indicate that the NGBM is ideally suitable for nonlinear systems including a class of approximate single-peak sequences [18,24,25,34-37].

Nevertheless, there are large stochastic fluctuation phenomena inevitably present in those real nonlinear systems due to uncertain external disturbances. The general NGBM cannot reveal the individual stochastic fluctuations but can only describe the integral single-peaked evolution tendency. Under such circumstances, it is impossible to ignore the apparent modeling errors by using the general NGBM. Therefore, it is very essential for exploring how to detect the individual stochastic fluctuations present in general evolution tendency of nonlinear systems with small samples.

\subsection{The self-memory algorithm}

Chaotic dynamics indicate that there are random behaviors exhibited even in simple dynamic systems due to nonlinearities; it also implies that a prediction method must consider randomness along with dynamics. The self-memory algorithm (SA) of dynamic systems has exactly filled the gap between determinism and randomness theory and has raised questions about the predictability of systems. As system change processes are often irreversible, SA was developed by introducing the memory function, which contains historical information, into the system dynamic equation. After defining the inner product in Hilbert space, the system dynamic equation (i.e., a differential equation) can be transformed into an appropriate difference-integral equation, which is termed as a self-memorization one. Then, the self-memorization equation can be used to predict the system status by means of 
discretization and estimating the memory coefficients. The original dynamic differential equation has the weakness of being sensitive to the initial value. Comparatively, the self-memorization equation can eliminate this drawback on account that it contains initial fields for multiple time points instead of initial fields at only a single time point. Accordingly, the prediction superiority of SA lies in that it not only merges dynamic calculations together with parameter estimation from historical data but also adequately extracts historical information from real systems in statistics.

The evolution process of system maybe described by a nonlinear function as follows:

$$
x(t+\Delta t)=f(\lambda, x(t)),
$$

where $x$ is a state variable in relation to time $t$, and $\lambda$ represents a set of all control parameters. Generally, $t_{0}, t_{1}, t_{2}, \cdots$ denote various moments, and $x_{0}, x_{1}, x_{2}, \cdots$ denote corresponding states. Let

$$
x_{n} \equiv x\left(t_{n}\right), t_{n}=t_{0}+n \Delta t,
$$

then Eq. (8) can be transformed into $x_{n+1}=f\left(\lambda, t, x_{n}\right)$. There are various kinds of external disturbances that result in system uncertainty. After adding a stochastic term that affects the system state, the system equation can be expressed as follows:

$$
\frac{\mathrm{d} x}{\mathrm{~d} t}=f(\lambda, x(t))+g(x) Q(t)
$$

where $g(x) Q(t)$ is multiplicative noise, $g Q(t)$ is additive noise and $g$ is a constant.

Assume that one time set $T=\left[t_{-p}, t_{-p+1}, \cdots, t_{-1}, t_{0}, t_{1}, \cdots, t_{q-1}, t_{q}\right]$, where $t_{-p}, t_{-p+1}, \cdots, t_{-1}$ denote historical time points (observation points) from $t_{-p}$ to $t_{-1}, t_{0}$ denotes the current initial time point (observation point), $t_{1}, \cdots, t_{q-1}, t_{q}$ denote the future forecasting time points (observation points) from $t_{1}$ to $t_{q}$. The spatial set is expressed as $R=\left[r_{\alpha}, \cdots, r_{i}, \cdots, r_{\beta}\right]$, where $r_{i}$ is the spatial point which is inspected. The inner product space $L^{2}: T \times R$, which is in the Hilbert space, can be defined as follows:

$$
(f, g)=\int_{a_{0}}^{b_{0}} f(\xi) g(\xi) \mathrm{d} \xi,\left(f, g \in L^{2}\right) .
$$

Generally, the system dynamics equation can be expressed as follows:

$$
\frac{\mathrm{d} x}{\mathrm{~d} t}=F(x, \lambda, t)
$$

where $x$ is a variable, $\lambda$ is a parameter, $t$ is time interval series, and $F(x, \lambda, t)$ is termed the dynamic kernel. Eq. (12) reflects the relationship between local change of $x$ at time $t$ and source function $F$. Then, the memory function is defined as $\beta(r, t)$, where $\beta$ is the function of $r$ and $t$, and $|\beta(r, t)| \leq 1$. If it is limited to spatial point $r_{i}$, the memory function can be simplified to $\beta(t)$.

The ordinary initial-value problem in the general NGBM corresponds to a Markov process where the next value depends only on the present value. While we make a prediction by SA, the memory function $\beta(t)$ possesses functions in memorizing $p+1$ values of $x$, because $p$ values before $t_{0}$ are always utilized. This is the algorithm mechanism for introducing a memory function $\beta(t)$ into the novel SA-NGBM.

\subsection{Self-memory algorithm-based nonlinear grey Bernoulli model (SA-NGBM)}

Based on the algorithms mentioned above, the SA prediction method is coupled with the general NGBM to construct a novel SA-NGBM. The modeling flowsheet of the SA-NGBM is displayed in Figure 1. The calculation was performed with the aid of the MATLAB program in order to decrease the computational complexity for model parameters, memory coefficients, and so on. 


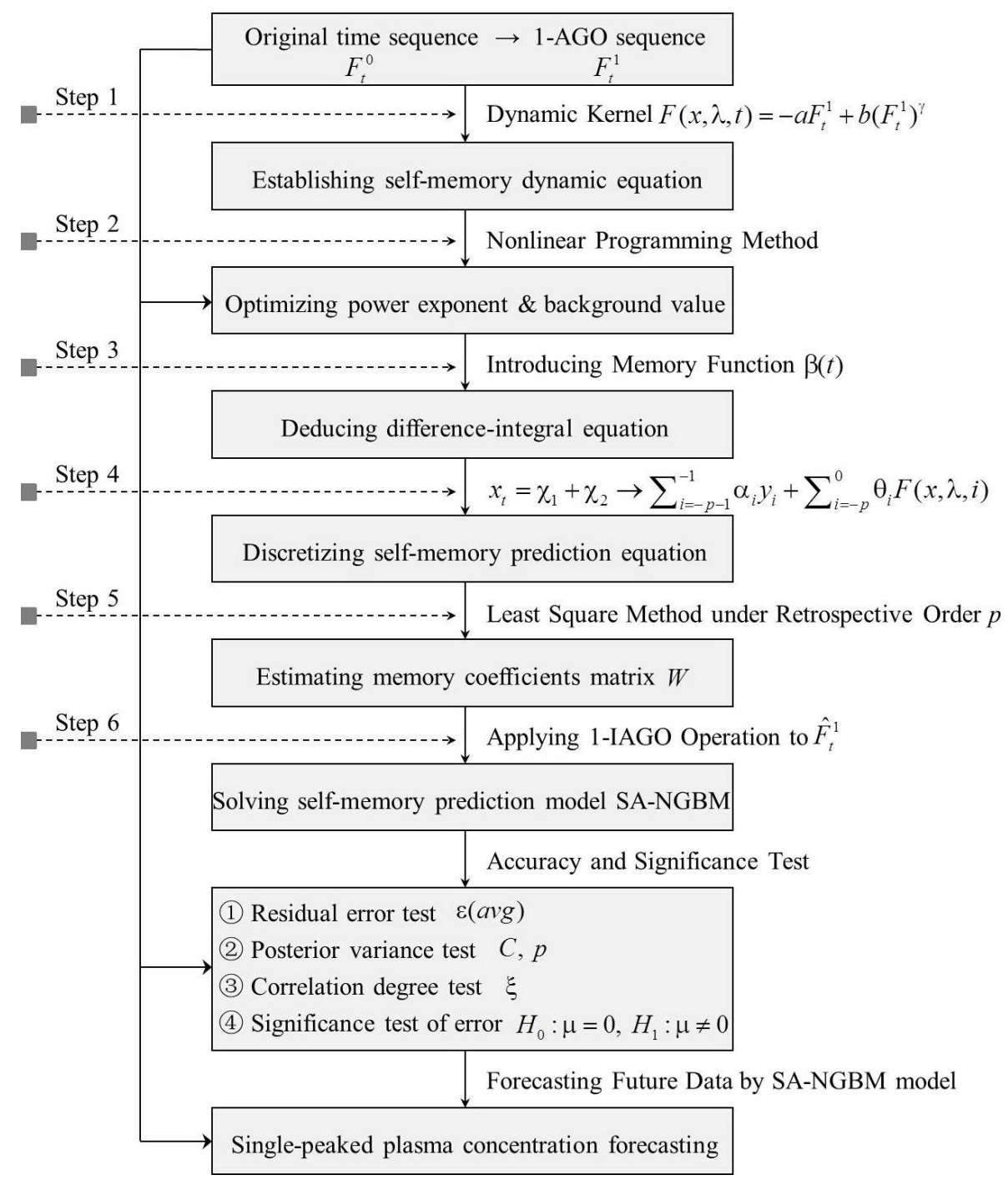

FIGURE 1 The modeling flowsheet of the SA-NGBM.

Considering nonlinear systems with small sample sizes, it is assumed that the original time sequence and the 1-AGO sequence are $F_{t}^{0}=\left\{f_{t}^{0} \mid t \in 1,2, \cdots, n\right\}$ and $F_{t}^{1}=\left\{f_{t}^{1} \mid t \in 1,2, \cdots, n\right\}$ respectively. Then, the coupling modeling procedure of the SA-NGBM is described as follows.

Step 1: Establishing the self-memory dynamic equation.

Let the differential equation $\frac{\mathrm{d} F_{t}^{1}}{\mathrm{~d} t}=-a F_{t}^{1}+b\left(F_{t}^{1}\right)^{\gamma}$, which is equal to the whitenization equation (5) of the NGBM, be $F(x, \lambda, t)=-a F_{t}^{1}+b\left(F_{t}^{1}\right)^{\gamma}$. Then, the self-memory dynamic equation of the novel SA-NGBM it is considered to be in the form $\frac{\mathrm{d} x}{\mathrm{~d} t}=F(x, \lambda, t)$, where the source function of dynamic kernel $F(x, \lambda, t)=-a F_{t}^{1}+b\left(F_{t}^{1}\right)^{\gamma}$.

Step 2: Optimizing the power exponent and background value.

The superiority of the NGBM lies in the fact that the adjustable value of power exponent $\gamma$, which matches with actual data, could be acquired by certain optimization techniques. Optimization occurs by supposing that the objective function is the minimization of mean absolute percentage error (MAPE) and that the constraint condition is the relationship among parameters $a, b$ and $\gamma$ [35-37]: 


$$
\begin{aligned}
& \underset{\gamma, \phi}{\operatorname{Min}} \operatorname{MAPE}=\frac{1}{n-1} \sum_{k=2}^{n}\left|\frac{\hat{f}_{t}^{0}-f_{t}^{0}}{f_{t}^{0}}\right| \\
& \text { S.T. }\left\{\begin{array}{l}
\gamma \neq 1 \\
0 \leq \phi \leq 1 \\
\hat{f}_{t}^{0}=\hat{f}_{t}^{1}-\hat{f}_{t-1}^{1} \\
\hat{f}_{t}^{1}=\left\{d+\left[\left(f_{1}^{0}\right)^{1-\gamma}-d\right] e^{-a(1-\gamma) t}\right\}^{1 / 1-\gamma} \\
d=\frac{\sum_{k=2}^{n}\left(g_{t}^{1}\right)^{2} \cdot \sum_{k=2}^{n} f_{t}^{0}\left(g_{t}^{1}\right)^{\gamma}-\sum_{k=2}^{n}\left(g_{t}^{1}\right)^{\gamma+1} \cdot \sum_{k=2}^{n} f_{t}^{0} g_{t}^{1}}{\sum_{k=2}^{n}\left(g_{t}^{1}\right)^{\gamma+1} \cdot \sum_{k=2}^{n} f_{t}^{0}\left(g_{t}^{1}\right)^{\gamma}-\sum_{k=2}^{n}\left(g_{t}^{1}\right)^{2 \gamma} \cdot \sum_{k=2}^{n} f_{t}^{0} g_{t}^{1}} \\
g_{t}^{1}=\phi \cdot f_{t}^{1}+(1-\phi) \cdot f_{t-1}^{1} \\
t=2,3, \cdots, n
\end{array}\right.
\end{aligned}
$$

The adjustable value of the power exponent $\gamma$ can be optimized by a nonlinear programming model (13). Meanwhile, the appropriate interpolated coefficient $\phi$, assumed to have a closed interval $[0,1]$, can be optimized against the background value $g_{t}^{1}=\phi \cdot f_{t}^{1}+(1-\phi) \cdot f_{t-1}^{1}$. This equation can be conveniently solved with the aid of LINGO software or MATLAB software, thereby obtaining the optimal power exponent $\gamma$ and the interpolated coefficient $\phi$.

Step 3: Deducing the difference-integral equation.

Assume that one time set $T=\left[t_{-p}, t_{-p+1}, \cdots, t_{-1}, t_{0}, t_{1}, \cdots, t_{q-1}, t_{q}\right]$, where the number $p$ of historical time points is the retrospective order of the system, and $q$ is the number of future forecasting time points. If considering the multiple historical time points $t_{-p}, t_{-p+1}, \cdots, t_{-1}$ and a single forecasting time point $t$, the memory function $\beta(t)$ and inner product operation (11) are introduced into $\frac{\mathrm{d} x}{\mathrm{~d} t}=-a F_{t}^{1}+b\left(F_{t}^{1}\right)^{\gamma}$. Meanwhile, the variable $x$ and memory function $\beta(t)$ are supposed to meet the conditions of continuous, differentiable and integrable. Then, the system dynamic equation can be changed to an analytic formula as follows:

$$
\int_{t_{-p}}^{t} \beta(\tau) \frac{\partial x}{\partial \tau} \mathrm{d} \tau=\int_{t_{-p}}^{t} \beta(\tau)\left[-a F_{\tau}^{1}+b\left(F_{\tau}^{1}\right)^{\gamma}\right] \mathrm{d} \tau
$$

which can be regarded as the weighted integral whose weight is memory coefficient $\beta(t)$. Eq. (14) then becomes

$$
\int_{t_{-p}}^{t_{-p+1}} \beta(\tau) \frac{\partial x}{\partial \tau} \mathrm{d} \tau+\int_{t_{-p+1}}^{t_{-p+2}} \beta(\tau) \frac{\partial x}{\partial \tau} \mathrm{d} \tau+\cdots+\int_{t_{0}}^{t} \beta(\tau) \frac{\partial x}{\partial \tau} \mathrm{d} \tau=\int_{t_{-p}}^{t} \beta(\tau)\left[-a F_{\tau}^{1}+b\left(F_{\tau}^{1}\right)^{\gamma}\right] \mathrm{d} \tau .
$$

The method of integration by parts and the mean value theorem of integrals are applied to all integral terms on the left-hand side of Eq. (15). After merging and eliminating the similar terms, a difference-integral equation is deduced as:

$$
\beta_{t} x_{t}-\beta_{-p} x_{-p}-\sum_{i=-p}^{0} x_{i}^{m}\left(\beta_{i+1}-\beta_{i}\right)-\int_{t_{-p}}^{t} \beta(\tau)\left[-a F_{\tau}^{1}+b\left(F_{\tau}^{1}\right)^{\gamma}\right] \mathrm{d} \tau=0
$$

where $\beta_{t} \equiv \beta(t), \quad x_{t} \equiv x(t), \quad \beta_{i} \equiv \beta\left(t_{i}\right), \quad x_{i} \equiv x\left(t_{i}\right), \quad i=-p,-p+1, \cdots, 0, \quad$ and mid-value $x_{i}^{m} \equiv x\left(t_{m}\right)$, $t_{i}<t_{m}<t_{i+1}$.

Step 4: Discretizing the self-memory prediction equation.

Let $x_{-p} \equiv x_{-p-1}^{m}$ and $\beta_{-p-1} \equiv 0$. Eq. (16) can then be transformed into

$$
x_{t}=\frac{1}{\beta_{t}} \sum_{i=-p-1}^{0} x_{i}^{m}\left(\beta_{i+1}-\beta_{i}\right)+\frac{1}{\beta_{t}} \int_{t_{-p}}^{t} \beta(\tau)\left[-a F_{\tau}^{1}+b\left(F_{\tau}^{1}\right)^{\gamma}\right] \mathrm{d} \tau=\chi_{1}+\chi_{2},
$$


and is termed the $p$-order self-memory prediction equation, where $p$ denotes the retrospective order. The self-memory equation (17) emphasizes that the predicted value $x_{t}$ at time point $t$ is the joint contribution of self-memory term $\chi_{1}$ and exogenous effect term $\chi_{2}$. Eq. (17) can be discretized by replacing the integral operation with summation, replacing the differential with a difference, and replacing the mid-value $x_{i}^{m}$ with the mean value at two contiguous time points, namely, $x_{i}^{m}=\frac{1}{2}\left(x_{i+1}+x_{i}\right) \equiv y_{i}$. The self-memory equation (17) can be discretized by taking the equidistance time interval $\Delta t_{i}=t_{i+1}-t_{i}=1$ for the time points, and merging $\beta_{t}$ with $\beta_{i}$ :

$$
x_{t}=\sum_{i=-p-1}^{-1} \alpha_{i} y_{i}+\sum_{i=-p}^{0} \theta_{i}\left[-a F_{i}^{1}+b\left(F_{i}^{1}\right)^{\gamma}\right]
$$

where $\alpha_{i}=\left(\beta_{i+1}-\beta_{i}\right) / \beta_{t}$ and $\theta_{i}=\beta_{i} / \beta_{t}$ are called memory coefficients.

Step 5: Estimating the memory coefficients matrix.

When compared to a general differential equation, there is an extra memory function $\beta(t)$ present in the self-memory equation. Therefore, the self-memory equation has no analytic solution, and the numerical solution only can be solved with the aid of a computer. If regarding $F$ and $x$ as the input and output of the systems respectively, the self-memory equation can be solved by using the ordinary least squares method (OLS). Assume that there is a historical data sequence at $L(L>p)$ time points, and OLS is used to estimate the memory coefficients $\alpha_{i}$ and $\theta_{i}$ in Eq. (18). Let

$$
\begin{aligned}
\underset{L \times 1}{X_{t}}=\left[\begin{array}{c}
x_{t 1} \\
x_{t 2} \\
\vdots \\
x_{t L}
\end{array}\right], \underset{L \times(p+1)}{Y}=\left[\begin{array}{cccc}
y_{-p-1,1} & y_{-p, 1} & \cdots & y_{-1,1} \\
y_{-p-1,2} & y_{-p, 2} & \cdots & y_{-1,2} \\
\vdots & \vdots & \ddots & \vdots \\
y_{-p-1, L} & y_{-p, L} & \cdots & y_{-1, L}
\end{array}\right], \quad \underset{(p+1) \times 1}{A}=\left[\begin{array}{c}
\alpha_{-p-1} \\
\alpha_{-p} \\
\vdots \\
\alpha_{-1}
\end{array}\right], \\
\underset{L \times(p+1)}{\Gamma}=\left[\begin{array}{cccc}
\left(-a F_{-p}^{1}+b\left(F_{-p}^{1}\right)^{\gamma}\right)_{1} & \left(-a F_{-p+1}^{1}+b\left(F_{-p+1}^{1}\right)^{\gamma}\right)_{1} & \cdots & \left(-a F_{0}^{1}+b\left(F_{0}^{1}\right)^{\gamma}\right)_{1} \\
\left(-a F_{-p}^{1}+b\left(F_{-p}^{1}\right)^{\gamma}\right)_{2} & \left(-a F_{-p+1}^{1}+b\left(F_{-p+1}^{1}\right)^{\gamma}\right)_{2} & \cdots & \left(-a F_{0}^{1}+b\left(F_{0}^{1}\right)^{\gamma}\right)_{2} \\
\vdots & \vdots & \ddots & \vdots \\
\left(-a F_{-p}^{1}+b\left(F_{-p}^{1}\right)^{\gamma}\right)_{L} & \left(-a F_{-p+1}^{1}+b\left(F_{-p+1}^{1}\right)^{\gamma}\right)_{L} & \cdots & \left(-a F_{0}^{1}+b\left(F_{0}^{1}\right)^{\gamma}\right)_{L}
\end{array}\right], \underset{(p+1) \times 1}{\Theta}=\left[\begin{array}{c}
\theta_{-p} \\
\theta_{-p+1} \\
\vdots \\
\theta_{0}
\end{array}\right],
\end{aligned}
$$

then the matrix form of Eq. (18) can be expressed as follows:

$$
X_{t}=Y A+\Gamma \Theta .
$$

If we let $Z=[Y, \Gamma], W=\left[\begin{array}{c}A \\ \Theta\end{array}\right]$, then Eq. (19) turns into $X_{t}=Z W$, and can thereby be described as the least square estimation of memory coefficients matrix $W$ is obtained by OLS:

$$
W=\left(Z^{\mathrm{T}} Z\right)^{-1} Z^{\mathrm{T}} X_{t}
$$

Step 6: Solving the self-memory prediction model.

After the memory coefficient matrix $W$ is substituted into the discretized prediction equation (18), the simulation and forecasting value $\hat{f}_{t}^{1}(\forall t \geq 1)$ of the 1-AGO sequence $F_{t}^{1}$ can be acquired. Then, by applying the 1-IAGO to $\hat{f}_{t}^{1}$, its corresponding inverse accumulated value $\hat{f}_{t}^{0}(\forall t \geq 1)$ can be solved as follows:

$$
\hat{f}_{t}^{0}=\hat{f}_{t}^{1}-\hat{f_{-1}^{1}}, \quad \forall t \geq 1,
$$

where $\hat{f}_{0}^{1} \equiv 0$. Consequently, the simulation and forecasting sequence $\hat{F}_{t}^{0}=\left\{\hat{f}_{t}^{0} \mid \forall t \geq 1\right\}$ of the original 
sequence $F_{t}^{0}$ is obtained.

\subsection{Accuracy and significance test of forecasting performance}

The purpose of prediction to provide services for decision making, and the credible and accurate predicting of outcomes are the precondition for scientific decision. Therefore, the accuracy and significance test results are important evaluation criteria for comparing the SA-NGBM with other prediction models. Only after the prediction model has passed through the accuracy and significance tests can it be used for extrapolation and application. In this study, four popular test indices for grey prediction methods have been used to compare the accuracy of different prediction models and test the corresponding significance as follows $[9,20]$.

Let $f_{t}^{0}(t \in 1,2, \cdots, n)$ denote the actual values at time point $t$ of the original sequence $F_{t}^{0}$, and $\hat{f}_{t}^{0}(t \in 1,2, \cdots, n)$ denote the corresponding predicted values. Thus $q(t)=f_{t}^{0}-\hat{f}_{t}^{0}(t \in 1,2, \cdots, n)$ is called the residual error at specific time point $t$. In addition, the RMSE $=\sqrt{\frac{1}{n} \sum_{t=1}^{n}\left(f_{t}^{0}-\hat{f}_{t}^{0}\right)^{2}}$ denotes the root mean square error.

Test criterion 1: Residual error test

The residual error test is one type of pointwise test method. The relative percentage error $\mathrm{RPE}_{t}$ at time point $t$ is defined as

$$
\operatorname{RPE}_{t}=\varepsilon(t)=\frac{q(t)}{f_{t}^{0}} \times 100 \%, t \in 2,3, \cdots, n,
$$

where $q(t)$ denotes the residual error at time point $t$. The average relative percentage error ARPE at all time points is expressed as

$$
\mathrm{ARPE}=\varepsilon(\operatorname{avg})=\frac{1}{n-1} \sum_{t=2}^{n}|\varepsilon(t)|, \quad t \in 2,3, \cdots, n .
$$

For a given $\alpha>0$, when $\mathrm{ARPE}<\alpha$ and $\mathrm{RPE}_{t}<\alpha$, then the model is supposed to pass the residual error test.

Test criterion 2: Posterior variance test

Let $\bar{f}=\frac{1}{n} \sum_{t=1}^{n} f_{t}^{0}$ and $S_{1}^{2}=\frac{1}{n} \sum_{t=1}^{n}\left(f_{t}^{0}-\bar{f}\right)^{2}$ denote the mean value and variance of the original sequence $F_{t}^{0}$, respectively. Moreover, $\bar{q}=\frac{1}{n} \sum_{t=1}^{n} q(t)$ and $S_{2}{ }^{2}=\frac{1}{n} \sum_{t=1}^{n}(q(t)-\bar{q})^{2}$ denote the mean value and variance of the residual error sequence $Q^{0}$, respectively. The ratio of mean square error $C=S_{2} / S_{1}$ is termed as the posterior variance ratio. For a given $C_{0}>0$, if there is $C<C_{0}$, then the model is a posterior-variance-ratio-qualified model. In the same way, for a given $p_{0}>0$, if there is $p=P\left\{|q(t)-\bar{q}|<0.6745 S_{1}\right\}>p_{0}$, then the model is a small-error-probability-qualified model. Accordingly, the model is considered to pass the posterior variance test.

Test criterion 3: Correlation degree test

Let $\xi=\frac{1+|\delta|+|\hat{\delta}|}{1+|\delta|+|\hat{\delta}|+|\hat{\delta}-\delta|}$ denote the absolute correlation degree between the original sequence $F_{t}^{0}$ and predicted sequence $\quad \hat{F}_{t}^{0} \quad, \quad$ where $\quad|\delta|=\left|\sum_{t=2}^{n-1} f_{t}^{0}+\frac{1}{2} f_{n}^{0}\right| \quad, \quad|\hat{\delta}|=\left|\sum_{t=2}^{n-1} \hat{f}_{t}^{0}+\frac{1}{2} \hat{f}_{n}^{0}\right| \quad$ and $|\hat{\delta}-\delta|=\left|\sum_{t=2}^{n-1}\left(\hat{f}_{t}^{0}-f_{t}^{0}\right)+\frac{1}{2}\left(\hat{f}_{n}^{0}-f_{n}^{0}\right)\right|$. For a given $\xi_{0}>0$, if the model satisfies $\xi_{>} \xi_{0}$, then it is regarded to 
pass the correlation degree test.

Test criterion 4: Significance test of error

Let $E_{t}=\frac{q(t)}{f_{t}^{0}}(t \in 2,3, \cdots, n)$, and it is generally considered to obey a normal distribution, i.e., $E_{t} \sim N\left(\mu, \sigma^{2}\right)$, where $\mu$ and $\sigma^{2}$ are unknown. The higher the prediction accuracy is, the closer to zero the mathematical expectation of relative error obeying a normal distribution is. Therefore, the level of prediction accuracy is equivalent to the hypothesis testing as follows:

$$
H_{0}: \mu=0, H_{1}: \mu \neq 0 \text {. }
$$

For a given confidence level $\alpha$, the test statistic $t(n-1)=\frac{\bar{E}}{s / \sqrt{n}}$ is constructed. If there is $|t(n-1)|>t_{1-\alpha / 2}$, then $H_{0}$ is rejected, meaning that there was a significant difference between the modeling error and zero. Conversely, if there is $|t(n-1)| \leq t_{1-\alpha / 2}$, then $H_{0}$ is accepted, meaning that there was no significant difference between them. Consequently, the model is considered to pass the significance test.

From the above, the residual error, posterior variance, correlation degree and significance can be used for testing the reliability and accuracy of prediction models. Four accuracy test indices for each model are comprehensively evaluated in Table 1.

TABLE 1 The commonly used scales of different test indices for accuracy test [9,20]

\begin{tabular}{lcccc}
\hline \multirow{2}{*}{ Accuracy class } & \multicolumn{4}{c}{ Accuracy test index } \\
\cline { 2 - 5 } & $\begin{array}{c}\text { Average relative error } \\
\varepsilon(\text { avg) }\end{array}$ & $\begin{array}{c}\text { Posterior variance ratio } \\
\text { 1st level (superior) }\end{array}$ & $\begin{array}{c}\text { Small error probability } \\
p\end{array}$ & $\begin{array}{c}\text { Correlation degree } \\
\xi\end{array}$ \\
\hline 2nd level (qualified) & $\leq 1 \%$ & $\leq 0.35$ & $\geq 95 \%$ & $\geq 0.90$ \\
3rd level (marginal) & $5 \% \sim 10 \%$ & $0.35 \sim 0.50$ & $80 \% \sim 95 \%$ & $0.80 \sim 0.90$ \\
4th level (disqualified) & $10 \% \sim 20 \%$ & $0.50 \sim 0.65$ & $70 \% \sim 80 \%$ & $0.70 \sim 0.80$ \\
\hline
\end{tabular}

\section{Empirical analysis on plasma concentration of acetylsalicylic acid}

\subsection{Data}

Plasma concentration data of acetylsalicylic acid were obtained from another study [38]. This study examined four experimental rabbits whose weights were between $2.5 \mathrm{~kg}$ and $3.0 \mathrm{~kg}$. After a 12 hour fast, they were given acetylsalicylic acid tablets $\left(50-100 \mathrm{mg} \cdot \mathrm{kg}^{-1}\right)$ orally. Then, the plasma concentration data at different time points after taking the medicine were obtained. Table 2 lists the acetylsalicylic acid plasma concentrations corresponding to those four experimental rabbits.

TABLE 2 Plasma concentration of acetylsalicylic acid of four groups [38] (unit: $\mu \mathrm{g} \cdot \mathrm{mL}^{-1}$ )

\begin{tabular}{ccccc}
\hline Time-point & Group 1 & Group 2 & Group 3 & Group 4 \\
\hline $1(0.15 \mathrm{~h})$ & 151.34 & 147.34 & 153.85 & 150.83 \\
\hline $2(0.25 \mathrm{~h})$ & 169.29 & 157.94 & 170.34 & 167.34 \\
\hline $3(0.50 \mathrm{~h})$ & 211.14 & 205.78 & 211.67 & 198.23 \\
\hline $4(1.00 \mathrm{~h})$ & 232.52 & 221.45 & 236.47 & 228.46 \\
\hline $5(1.50 \mathrm{~h})$ & 247.01 & 250.63 & 258.65 & 249.85 \\
\hline$(2.00 \mathrm{~h})$ & 252.33 & 259.85 & 266.24 & 261.85 \\
$7(2.50 \mathrm{~h})$ & 255.87 & 264.46 & 273.45 & 270.46 \\
\hline $8(3.00 \mathrm{~h})$ & 258.42 & 269.74 & 277.46 & 275.27 \\
$9(3.50 \mathrm{~h})$ & 231.21 & 249.83 & 257.53 & 252.99 \\
$10(4.00 \mathrm{~h})$ & 211.85 & 228.73 & 237.35 & 230.53 \\
\hline
\end{tabular}




\begin{tabular}{lcccc}
$11(6.00 \mathrm{~h})$ & 170.36 & 192.35 & 209.35 & 201.75 \\
\hline $12(13.0 \mathrm{~h})$ & 126.55 & 143.63 & 151.43 & 149.64 \\
$13(22.0 \mathrm{~h})$ & 93.43 & 106.42 & 111.34 & 109.25 \\
\hline
\end{tabular}

Testing the significance of differences between the four groups of plasma time-concentration data should be carried out before conducting prediction. Above all, Friedman rank sum testing was conducted for the four groups of data as a whole. The Friedman chi-squared was 47.473, and the p-value was far less than 0.00001. Results indicate that there existed significant differences and high correlations among the four experimental groups. Wilcoxon signed-rank testing was then employed on each data pair. Testing results showed that there existed significant differences between each two experimental groups, except between group 1 and group 2. Therefore, the four groups of plasma time-concentration data have passed the significance testing.

Figure 2 shows the four groups of plasma concentration trend curves of acetylsalicylic acid. As is seen in this figure, there was an obvious single-peak tendency for each group of plasma concentrations, accompanied by irregular fluctuations due to the unstable changes occurring from the experimental and material factors. In conclusion, the plasma concentrations of acetylsalicylic acid showed an obvious nonlinear characteristic of single-peak tendency with stochastic fluctuations.
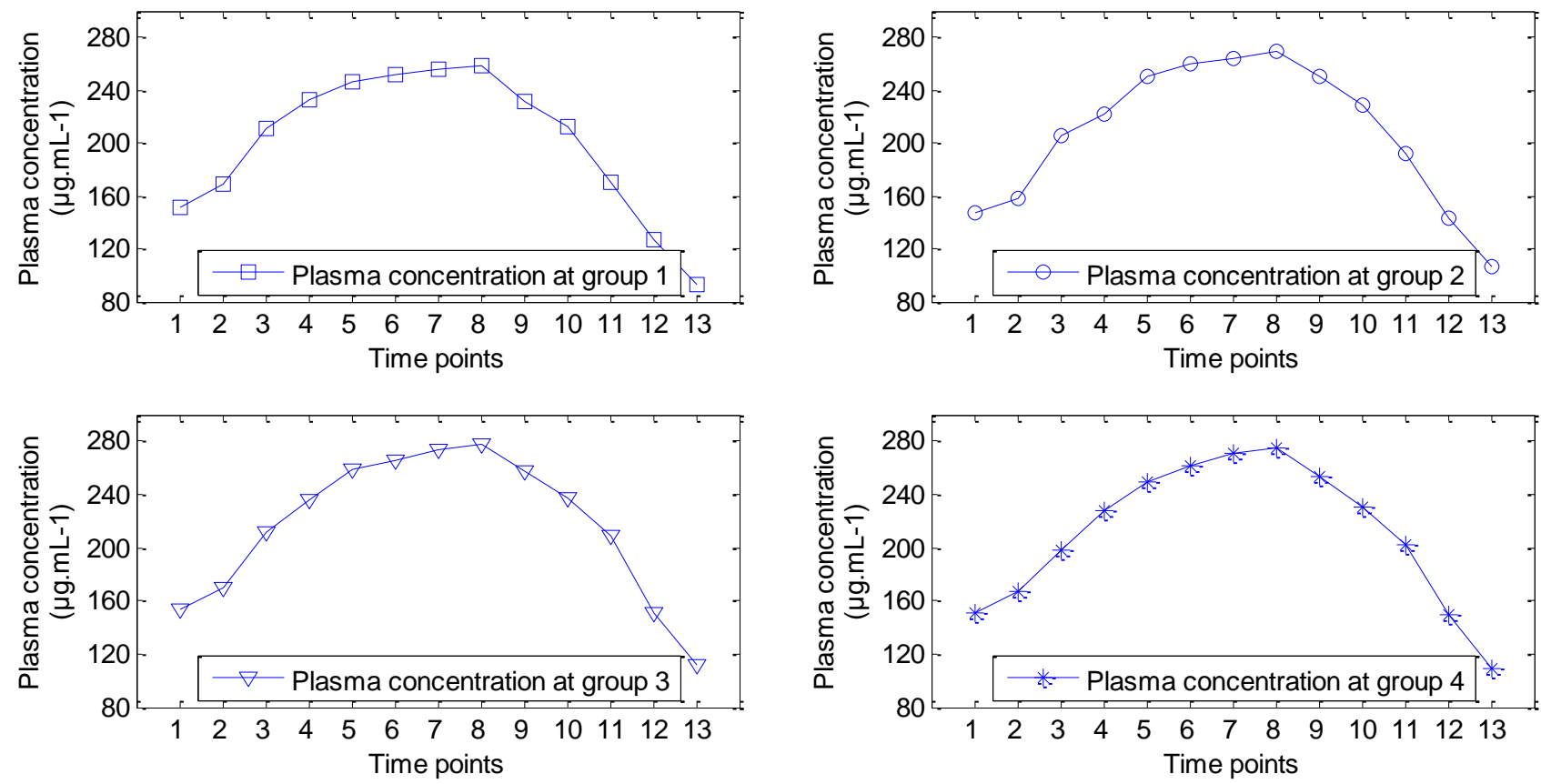

FIGURE 2 Plasma concentration tendency of acetylsalicylic acid of four groups.

\subsection{Results}

As a result of factors introducing uncertainty, such as the precision of detection and experimental error, the plasma concentrations of acetylsalicylic acid present typical grey system characteristics with small sample sizes and poor information. For this reason, this paper will build the SA-NGBM, an optimized NGBM (ONGBM) [36], an exponential smoothing model (ES) [39] and a simple moving average model (SMA) [40] to simulate the plasma concentration of acetylsalicylic acid at different time points. The plasma concentration data of the first eleven time points are taken as the simulation samples, and the data of the latter two time points are chosen as testing samples for prediction testing. Meanwhile, four popular accuracy and significance test criteria are adopted to check the prediction accuracy of different prediction models.

3.2.1. Forecasting the plasma concentration of acetylsalicylic acid of group 1

Following the modeling steps to couple the SA-NGBM with the SA algorithm, the power exponent is $\gamma=0.573411$, the interpolated coefficient of background value is $\phi=0.594408$, and the corresponding values 
of the parameter $a, b$ are $0.313723,10.804450$, respectively. Meanwhile, the value of the retrospective order is determined as $p=2$ by trial calculation method by minimizing the root-mean-square error. The memory coefficients are $\alpha_{-3}=0.403296, \alpha_{-2}=-1.846963, \alpha_{-1}=2.395267, \theta_{-2}=0.414322, \theta_{-1}=0.251846$ and $\theta_{0}=0.040747$, respectively. The model values of the SA-NGBM and other three compared models are shown in Table 3. There are no simulated values of the first two time points owing to the retrospective order $p=2$ in the SA-NGBM.

TABLE 3 The predicted values for plasma concentration of acetylsalicylic acid of group 1 (unit: $\mu \mathrm{g} \cdot \mathrm{mL}^{-1}$ )

\begin{tabular}{|c|c|c|c|c|c|c|c|c|c|}
\hline \multirow[t]{2}{*}{$\begin{array}{l}\text { Time- } \\
\text { point }\end{array}$} & \multirow{2}{*}{$\begin{array}{c}\text { Plasma } \\
\text { concentration } \\
\text { at group } 1 \\
f_{t}^{0}\end{array}$} & \multicolumn{2}{|c|}{$\begin{array}{l}\text { SA-NGBM model } \\
\gamma=0.573411 \\
\phi=0.594408, p=2\end{array}$} & \multicolumn{2}{|c|}{$\begin{array}{l}\text { ONGBM model } \\
\gamma=0.595842 \\
\phi=0.5\end{array}$} & \multicolumn{2}{|c|}{$\begin{array}{l}\text { Single exponential } \\
\text { smoothing }\end{array}$} & \multicolumn{2}{|c|}{ Simple moving average } \\
\hline & & $\hat{f}_{t}^{0}$ & $\varepsilon(t)$ & $\hat{f}_{t}^{0}$ & $\varepsilon(t)$ & $\hat{f}_{t}^{0}$ & $\varepsilon(t)$ & $\hat{f}_{t}^{0}$ & $\varepsilon(t)$ \\
\hline 2 & 169.29 & - & - & 169.290 & $0.000 \%$ & 169.290 & $0.000 \%$ & - & - \\
\hline 3 & 211.14 & - & - & 212.093 & $-0.451 \%$ & 169.290 & $19.821 \%$ & - & - \\
\hline 4 & 232.52 & 232.593 & $-0.031 \%$ & 238.766 & $-2.686 \%$ & 206.955 & $10.995 \%$ & - & - \\
\hline 5 & 247.01 & 244.099 & $1.178 \%$ & 251.908 & $-1.983 \%$ & 229.964 & $6.901 \%$ & 204.317 & $17.284 \%$ \\
\hline 6 & 252.33 & 259.987 & $-3.035 \%$ & 254.530 & $-0.872 \%$ & 245.305 & $2.784 \%$ & 230.223 & $8.761 \%$ \\
\hline 7 & 255.87 & 254.026 & $0.721 \%$ & 249.426 & $2.518 \%$ & 251.628 & $1.658 \%$ & 243.953 & $4.657 \%$ \\
\hline 8 & 258.42 & 246.525 & $4.603 \%$ & 238.955 & $7.532 \%$ & 255.446 & $1.151 \%$ & 251.737 & $2.586 \%$ \\
\hline 9 & 231.21 & 244.214 & $-5.624 \%$ & 225.007 & $2.683 \%$ & 258.123 & $11.640 \%$ & 255.540 & $-10.523 \%$ \\
\hline 10 & 211.85 & 206.735 & $2.414 \%$ & 209.035 & $1.329 \%$ & 233.901 & $10.409 \%$ & 248.500 & $-17.300 \%$ \\
\hline \multirow[t]{2}{*}{11} & 170.36 & 172.230 & $-1.098 \%$ & 192.124 & $-12.775 \%$ & 214.055 & $25.649 \%$ & 233.827 & $-37.255 \%$ \\
\hline & $\varepsilon(a v g)$ & & $2.338 \%$ & & $3.283 \%$ & & $9.101 \%$ & & $14.052 \%$ \\
\hline 12 & 126.55 & 137.129 & $-8.360 \%$ & 175.058 & $-38.331 \%$ & 174.730 & $-38.072 \%$ & 204.473 & $-61.575 \%$ \\
\hline 13 & 93.43 & 79.610 & $14.792 \%$ & 128.968 & $-38.037 \%$ & 131.368 & $-40.606 \%$ & 169.587 & $-81.512 \%$ \\
\hline
\end{tabular}

The accuracy and significance test criteria values of different prediction models for plasma concentration of acetylsalicylic acid of group 1 are listed in Table 4.

TABLE 4 Accuracy and significance test of predicted values for different grey prediction models

\begin{tabular}{lcccccc}
\hline \multicolumn{1}{c}{ Model } & RMSE & $\varepsilon($ avg $)$ & $C$ & $p$ & $\xi$ & Significance $(\alpha=0.95)$ \\
\hline SA-NGBM model & 7.165581 & $2.338 \%$ & 0.259230 & $100.000 \%$ & 0.9896 & significant \\
ONGBM model & 10.046072 & $3.283 \%$ & 0.320229 & $90.000 \%$ & 0.9911 & significant \\
Single exponential smoothing & 24.272530 & $9.101 \%$ & 0.773521 & $50.000 \%$ & 0.9745 & nonsignificant \\
Simple moving average & 34.767227 & $14.052 \%$ & 1.159815 & $42.857 \%$ & 0.6435 & nonsignificant \\
\hline
\end{tabular}

\subsubsection{Forecasting the plasma concentration of acetylsalicylic acid of group 2}

In the same way as group 1 , the power exponent $\gamma=0.631898$, background value $\phi=0.527600$, and parameter $a=0.365241, b=7.925877$ are obtained for group 2. Meanwhile, under the retrospective order $p=2$, the corresponding memory coefficients are $\alpha_{-3}=0.109115, \alpha_{-2}=-1.580079, \alpha_{-1}=2.419673$, $\theta_{-2}=-0.066091, \theta_{-1}=0.302783$ and $\theta_{0}=0.177644$, respectively. The model values of the SA-NGBM and other three compared models are shown in Table 5. Similarly, there are no simulated values of the first two time points in the SA-NGBM.

TABLE 5 The predicted values for plasma concentration of acetylsalicylic acid of group 2 (unit: $\mu \mathrm{g} \cdot \mathrm{mL}^{-1}$ )

\begin{tabular}{ccllll}
\hline Time- & Plasma & SA-NGBM model & ONGBM model & Single exponential & Simple moving average \\
point & concentration & $\gamma=0.631898$ & $\gamma=0.639316$ & smoothing & \\
& at group 2 & $\phi=0.527600, p=2$ & $\phi=0.5$ & & \\
\cline { 3 - 5 } & & &
\end{tabular}




\begin{tabular}{cccccccccc} 
& $f_{t}^{0}$ & $\hat{f}_{t}^{0}$ & $\varepsilon(t)$ & $\hat{f}_{t}^{0}$ & $\varepsilon(t)$ & $\hat{f}_{t}^{0}$ & $\varepsilon(t)$ & $\hat{f}_{t}^{0}$ & $\varepsilon(t)$ \\
\hline 2 & 157.94 & - & - & 157.940 & $0.000 \%$ & 157.940 & $0.000 \%$ & - & - \\
3 & 205.78 & - & - & 202.751 & $1.472 \%$ & 157.940 & $23.248 \%$ & - & - \\
4 & 221.45 & 221.054 & $0.179 \%$ & 234.062 & $-5.695 \%$ & 200.996 & $9.236 \%$ & - & - \\
5 & 250.63 & 250.475 & $0.062 \%$ & 252.688 & $-0.821 \%$ & 219.405 & $12.459 \%$ & 195.057 & $22.173 \%$ \\
\hline 6 & 259.85 & 260.314 & $-0.179 \%$ & 260.579 & $-0.281 \%$ & 247.507 & $4.750 \%$ & 225.953 & $13.045 \%$ \\
7 & 264.46 & 271.012 & $-2.478 \%$ & 259.983 & $1.693 \%$ & 258.616 & $2.210 \%$ & 243.977 & $7.745 \%$ \\
8 & 269.74 & 256.322 & $4.974 \%$ & 253.049 & $6.188 \%$ & 263.876 & $2.174 \%$ & 258.313 & $4.236 \%$ \\
\hline 9 & 249.83 & 256.739 & $-2.765 \%$ & 241.646 & $3.276 \%$ & 269.154 & $-7.735 \%$ & 264.683 & $-5.945 \%$ \\
10 & 228.73 & 229.996 & $-0.553 \%$ & 227.315 & $0.619 \%$ & 251.762 & $-10.070 \%$ & 261.343 & $-14.258 \%$ \\
11 & 192.35 & 191.453 & $0.466 \%$ & 211.275 & $-9.839 \%$ & 231.033 & $-20.111 \%$ & 249.433 & $-29.677 \%$ \\
\hline 12 & $\varepsilon($ avg & & & $1.457 \%$ & & $2.988 \%$ & & $9.199 \%$ & $13.869 \%$ \\
\hline 13 & 143.63 & 153.590 & $-6.935 \%$ & 194.456 & $-35.387 \%$ & 196.218 & $-36.614 \%$ & 223.637 & $-55.704 \%$ \\
\hline
\end{tabular}

The accuracy and significance test criteria values of different prediction models for plasma concentration of acetylsalicylic acid of group 2 are listed in Table 6.

TABLE 6 Accuracy and significance test of predicted values for different grey prediction models

\begin{tabular}{lcccccc}
\hline \multicolumn{1}{c}{ Model } & RMSE & $\varepsilon(a v g)$ & $C$ & $p$ & $\xi$ & Significance $(\alpha=0.95)$ \\
\hline SA-NGBM model & 5.847106 & $1.457 \%$ & 0.238907 & $100.000 \%$ & 0.9898 & significant \\
ONGBM model & 9.480363 & $2.988 \%$ & 0.276589 & $100.000 \%$ & 0.9937 & significant \\
Single exponential smoothing & 25.106145 & $9.199 \%$ & 0.721896 & $50.000 \%$ & 0.9561 & nonsignificant \\
Simple moving average & 36.508674 & $13.869 \%$ & 1.469318 & $14.286 \%$ & 0.5166 & nonsignificant \\
\hline
\end{tabular}

3.2.3. Forecasting the plasma concentration of acetylsalicylic acid of group 3

In the same way as groups 1 and 2 , the power exponent $\gamma=0.582311$, background value $\phi=0.550247$, and parameter $a=0.290872, b=9.948943$ are obtained. Meanwhile, under the retrospective order $p=2$, the corresponding memory coefficients are $\alpha_{-3}=0.341151, \alpha_{-2}=-0.934582, \alpha_{-1}=1.570336$, $\theta_{-2}=-7.756000, \theta_{-1}=18.737145$ and $\theta_{0}=-9.831399$, respectively. The model values of the SA-NGBM and other three compared models are shown in Table 7. Similarly, there are no simulated values of the first two time points in the SA-NGBM.

TABLE 7 The predicted values for plasma concentration of acetylsalicylic acid of group 3 (unit: $\mu \mathrm{g} \cdot \mathrm{mL}^{-1}$ )

\begin{tabular}{|c|c|c|c|c|c|c|c|c|c|}
\hline \multirow[t]{2}{*}{$\begin{array}{l}\text { Time- } \\
\text { point }\end{array}$} & \multirow{2}{*}{$\begin{array}{c}\text { Plasma } \\
\text { concentration } \\
\text { at group } 3 \\
f_{t}^{0}\end{array}$} & \multicolumn{2}{|c|}{$\begin{array}{l}\text { SA-NGBM model } \\
\gamma=0.582311 \\
\phi=0.550247, p=2\end{array}$} & \multicolumn{2}{|c|}{$\begin{array}{l}\text { ONGBM model } \\
\gamma=0.607507 \\
\phi=0.5\end{array}$} & \multicolumn{2}{|c|}{$\begin{array}{l}\text { Single exponential } \\
\text { smoothing }\end{array}$} & \multicolumn{2}{|c|}{ Simple moving average } \\
\hline & & $\hat{f}_{t}^{0}$ & $\varepsilon(t)$ & $\hat{f}_{t}^{0}$ & $\varepsilon(t)$ & $\hat{f}_{t}^{0}$ & $\varepsilon(t)$ & $\hat{f}_{t}^{0}$ & $\varepsilon(t)$ \\
\hline 2 & 170.34 & - & - & 166.534 & $2.234 \%$ & 170.340 & $0.000 \%$ & - & - \\
\hline 3 & 211.67 & - & - & 211.670 & $0.000 \%$ & 170.340 & $19.526 \%$ & - & - \\
\hline 4 & 236.47 & 236.358 & $0.047 \%$ & 242.731 & $-2.648 \%$ & 207.537 & $12.235 \%$ & - & - \\
\hline 5 & 258.65 & 257.480 & $0.452 \%$ & 261.155 & $-0.968 \%$ & 233.577 & $9.694 \%$ & 206.160 & $20.294 \%$ \\
\hline 6 & 266.24 & 269.010 & $-1.040 \%$ & 269.123 & $-1.083 \%$ & 256.143 & $3.792 \%$ & 235.597 & $11.510 \%$ \\
\hline 7 & 273.45 & 276.333 & $-1.054 \%$ & 268.902 & $1.663 \%$ & 265.230 & $3.006 \%$ & 253.787 & $7.191 \%$ \\
\hline 8 & 277.46 & 266.057 & $4.110 \%$ & 262.561 & $5.370 \%$ & 272.628 & $1.742 \%$ & 266.113 & $4.090 \%$ \\
\hline 9 & 257.53 & 267.177 & $-3.746 \%$ & 251.864 & $2.200 \%$ & 276.977 & $-7.551 \%$ & 272.383 & $-5.767 \%$ \\
\hline 10 & 237.35 & 233.946 & $1.434 \%$ & 238.254 & $-0.381 \%$ & 259.475 & $-9.322 \%$ & 269.480 & $-13.537 \%$ \\
\hline
\end{tabular}




\begin{tabular}{rrrrrrrrrr}
11 & 209.35 & 210.872 & $-0.727 \%$ & 222.870 & $-6.458 \%$ & 239.562 & $-14.431 \%$ & 257.447 & $-22.974 \%$ \\
\hline 12 & $\varepsilon($ avg $)$ & & $1.576 \%$ & & $2.301 \%$ & & $8.130 \%$ & & $12.195 \%$ \\
\hline 13 & 151.43 & 183.598 & $-21.243 \%$ & 206.587 & $-36.424 \%$ & 212.371 & $-40.244 \%$ & 234.743 & $-55.017 \%$ \\
\hline
\end{tabular}

The accuracy and significance test criteria values of different prediction models for plasma concentration of acetylsalicylic acid at group 3 are listed in Table 8 .

TABLE 8 Accuracy and significance test of predicted values for different grey prediction models

\begin{tabular}{lcccccc}
\hline \multicolumn{1}{c}{ Model } & RMSE & $\varepsilon(a v g)$ & $C$ & $p$ & $\xi$ & Significance $(\alpha=0.95)$ \\
\hline SA-NGBM model & 5.638736 & $1.576 \%$ & 0.263565 & $100.000 \%$ & 0.9971 & significant \\
ONGBM model & 7.257053 & $2.301 \%$ & 0.224003 & $100.000 \%$ & 0.9811 & significant \\
Single exponential smoothing & 22.687607 & $8.130 \%$ & 0.685827 & $50.000 \%$ & 0.9543 & nonsignificant \\
Simple moving average & 33.329205 & $12.195 \%$ & 1.511051 & $14.286 \%$ & 0.5109 & nonsignificant \\
\hline
\end{tabular}

\subsubsection{Forecasting the plasma concentration of acetylsalicylic acid of group 4}

In the same way as the prior groups, the power exponent $\gamma=0.665516$, background value $\phi=0.607350$, and parameter $a=0.416486, b=6.833010$ are obtained. Meanwhile, under the retrospective order $p=2$, the corresponding memory coefficients are $\alpha_{-3}=-0.082427, \alpha_{-2}=-0.518745, \alpha_{-1}=1.572655$, $\theta_{-2}=-5.859309, \theta_{-1}=13.065967$ and $\theta_{0}=-6.406394$, respectively. The model values of the SA-NGBM and other three compared models are shown in Table 9. Similarly, there are no simulated values of the first two time points in the SA-NGBM.

TABLE 9 The predicted values for plasma concentration of acetylsalicylic acid of group 4 (unit: $\mu \mathrm{g} \cdot \mathrm{mL}^{-1}$ )

\begin{tabular}{|c|c|c|c|c|c|c|c|c|c|}
\hline \multirow[t]{2}{*}{$\begin{array}{l}\text { Time- } \\
\text { point }\end{array}$} & \multirow{2}{*}{$\begin{array}{c}\text { Plasma } \\
\text { concentration } \\
\text { at group } 4 \\
f_{t}^{0}\end{array}$} & \multicolumn{2}{|c|}{$\begin{array}{l}\text { SA-NGBM model } \\
\gamma=0.665516 \\
\phi=0.607350, p=2\end{array}$} & \multicolumn{2}{|c|}{$\begin{array}{l}\text { ONGBM model } \\
\gamma=0.716306 \\
\phi=0.5\end{array}$} & \multicolumn{2}{|c|}{$\begin{array}{l}\text { Single exponential } \\
\text { smoothing }\end{array}$} & \multicolumn{2}{|c|}{ Simple moving average } \\
\hline & & $\hat{f}_{t}^{0}$ & $\varepsilon(t)$ & $\hat{f}_{t}^{0}$ & $\varepsilon(t)$ & $\hat{f}_{t}^{0}$ & $\varepsilon(t)$ & $\hat{f}_{t}^{0}$ & $\varepsilon(t)$ \\
\hline 2 & 167.34 & - & - & 151.319 & $9.574 \%$ & 167.340 & $0.000 \%$ & - & - \\
\hline 3 & 198.23 & - & - & 198.230 & $0.000 \%$ & 167.340 & $15.583 \%$ & - & - \\
\hline 4 & 228.46 & 228.366 & $0.041 \%$ & 233.284 & $-2.112 \%$ & 195.141 & $14.584 \%$ & - & - \\
\hline 5 & 249.85 & 247.001 & $1.140 \%$ & 255.542 & $-2.278 \%$ & 225.128 & $9.895 \%$ & 198.010 & $20.748 \%$ \\
\hline 6 & 261.85 & 269.918 & $-3.081 \%$ & 266.057 & $-1.607 \%$ & 247.378 & $5.527 \%$ & 225.513 & $13.877 \%$ \\
\hline 7 & 270.46 & 268.270 & $0.810 \%$ & 266.806 & $1.351 \%$ & 260.403 & $3.718 \%$ & 246.720 & $8.778 \%$ \\
\hline 8 & 275.27 & 264.234 & $4.009 \%$ & 260.027 & $5.537 \%$ & 269.454 & $2.113 \%$ & 260.720 & $5.286 \%$ \\
\hline 9 & 252.99 & 262.799 & $-3.877 \%$ & 247.852 & $2.031 \%$ & 274.688 & $-8.577 \%$ & 269.193 & $-6.405 \%$ \\
\hline 10 & 230.53 & 230.290 & $0.104 \%$ & 232.124 & $-0.691 \%$ & 255.160 & $-10.684 \%$ & 266.240 & $-15.490 \%$ \\
\hline 11 & 201.75 & 200.155 & $0.791 \%$ & 214.339 & $-6.240 \%$ & 232.993 & $-15.486 \%$ & 252.930 & $-25.368 \%$ \\
\hline & $\varepsilon(a v g)$ & & $1.732 \%$ & & $3.142 \%$ & & $8.617 \%$ & & $13.707 \%$ \\
\hline 12 & 149.64 & 169.648 & $-13.371 \%$ & 195.654 & $-30.750 \%$ & 204.874 & $-36.911 \%$ & 228.423 & $-52.648 \%$ \\
\hline 13 & 109.25 & 106.204 & $2.788 \%$ & 152.553 & $-39.637 \%$ & 155.163 & $-42.026 \%$ & 193.973 & $-77.550 \%$ \\
\hline
\end{tabular}

The accuracy and significance test criteria values of different prediction models for plasma concentration of acetylsalicylic acid at group 3 are listed in Table 10.

TABLE 10 Accuracy and significance test of predicted values for different grey prediction models

\begin{tabular}{lcccccc}
\hline \multicolumn{1}{c}{ Model } & RMSE & $\varepsilon($ avg $)$ & $C$ & $p$ & $\xi$ & Significance $(\alpha=0.95)$ \\
\hline SA-NGBM model & 6.109683 & $1.732 \%$ & 0.264338 & $100.000 \%$ & 0.9957 & significant \\
ONGBM model & 8.735930 & $3.142 \%$ & 0.258615 & $100.000 \%$ & 0.9138 & significant
\end{tabular}




\begin{tabular}{lllllll} 
Single exponential smoothing & 22.524427 & $8.617 \%$ & 0.660678 & $50.000 \%$ & 0.9557 & nonsignificant \\
Simple moving average & 35.737509 & $13.707 \%$ & 1.506252 & $14.286 \%$ & 0.5299 & nonsignificant \\
\hline
\end{tabular}

\subsection{Discussion}

From the viewpoint of accuracy and significance testing, the nonlinear grey Bernoulli model is particularly appropriate to approximate single-peak sequences. It is shown that the NGBM can take into full consideration the characteristics of approximating single-peaks among sequences and is able to adequately reflect the integral evolution laws of nonlinear systems compared with the ES [39] and SMA [40] models. Nevertheless, as a result of uncertainty about external disturbances, the traditional NGBM $[24,25]$ cannot effectively reflect large stochastic fluctuations that commonly exist in nonlinear systems. In contrast, the SA method helped the NGBM to closely catch individual stochastic variations. The limitations of the traditional NGBM, such as sensitivity to initial values of the differential equation, can be eliminated by using multi-timepoint initial fields instead using data from only a single time point [30]. The prediction accuracy of the SA-NGBM was further improved compared with the traditional ONGBM. Meanwhile, the fitting curves of plasma concentration of acetylsalicylic acid and their corresponding comparison results of the $\mathrm{RPE}_{t}$ distribution are shown in Figures 3-6 respectively. As seen from the following figures, the proposed SA-NGBM model can better represent the tendency of integral single-peak development, along with individual fluctuations existing in nonlinear systems, and possesses more stable and ideal prediction effects. Therefore, it is a reliable and stable model for predicting the future evolutionary trend of single-peaked nonlinear systems with small sample sizes.
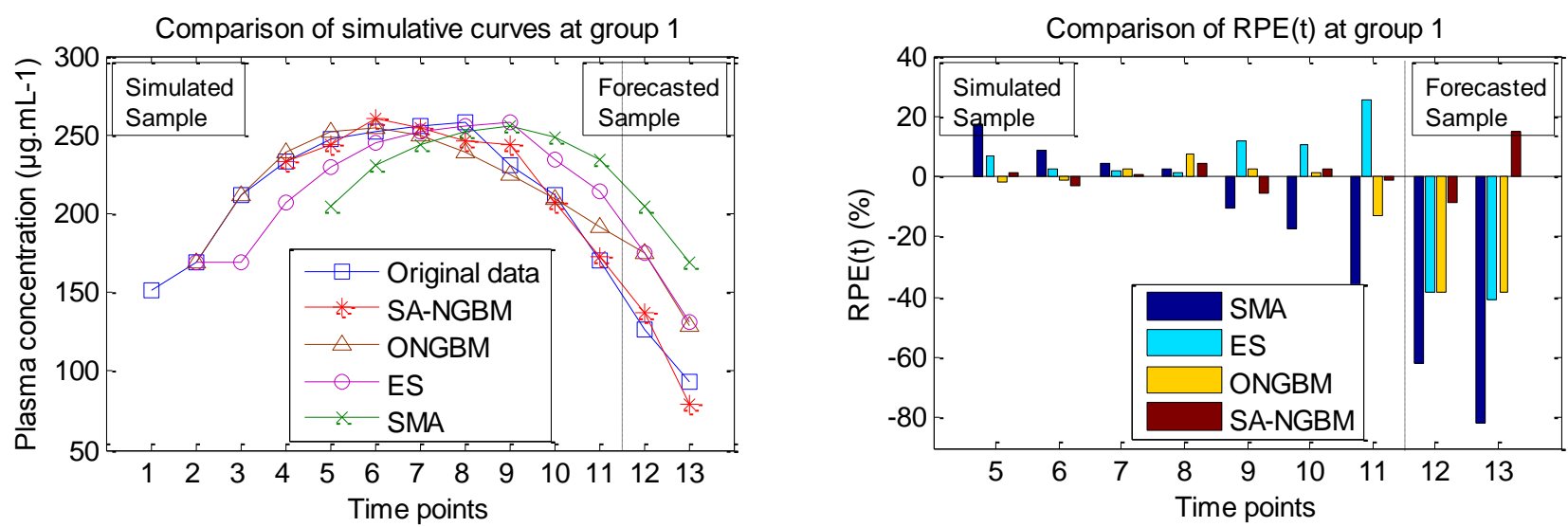

FIGURE 3 Comparison among original and simulative curves of different prediction models of group 1 .
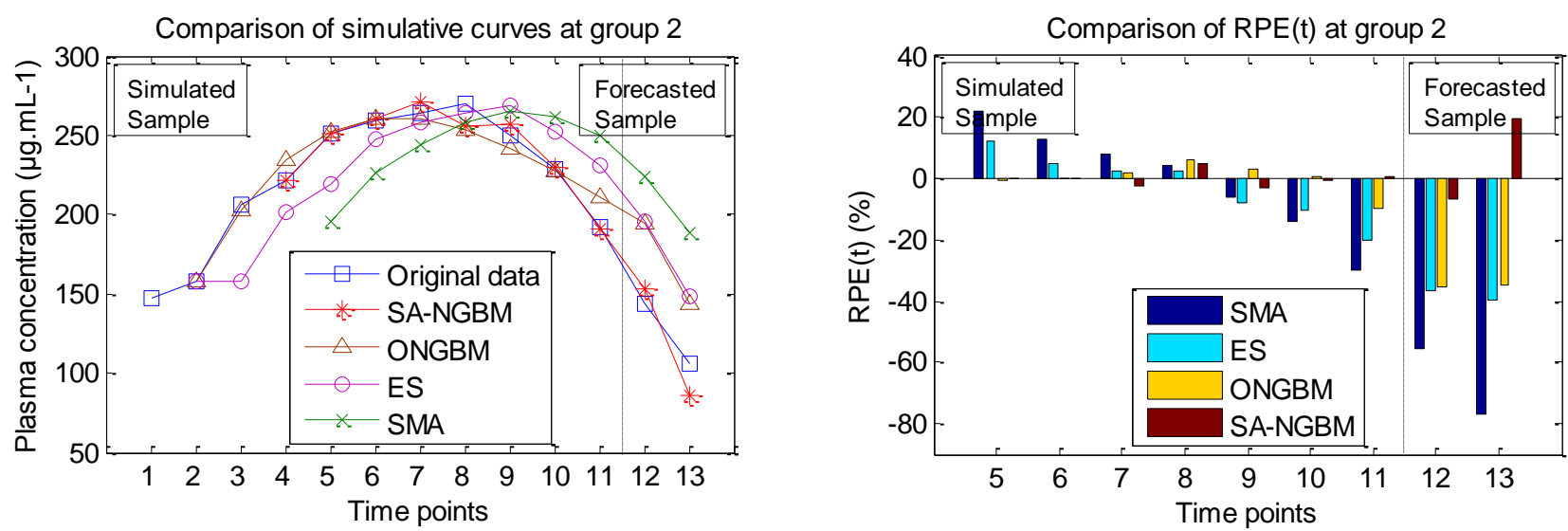

FIGURE 4 Comparison among original and simulative curves of different prediction models of group 2. 

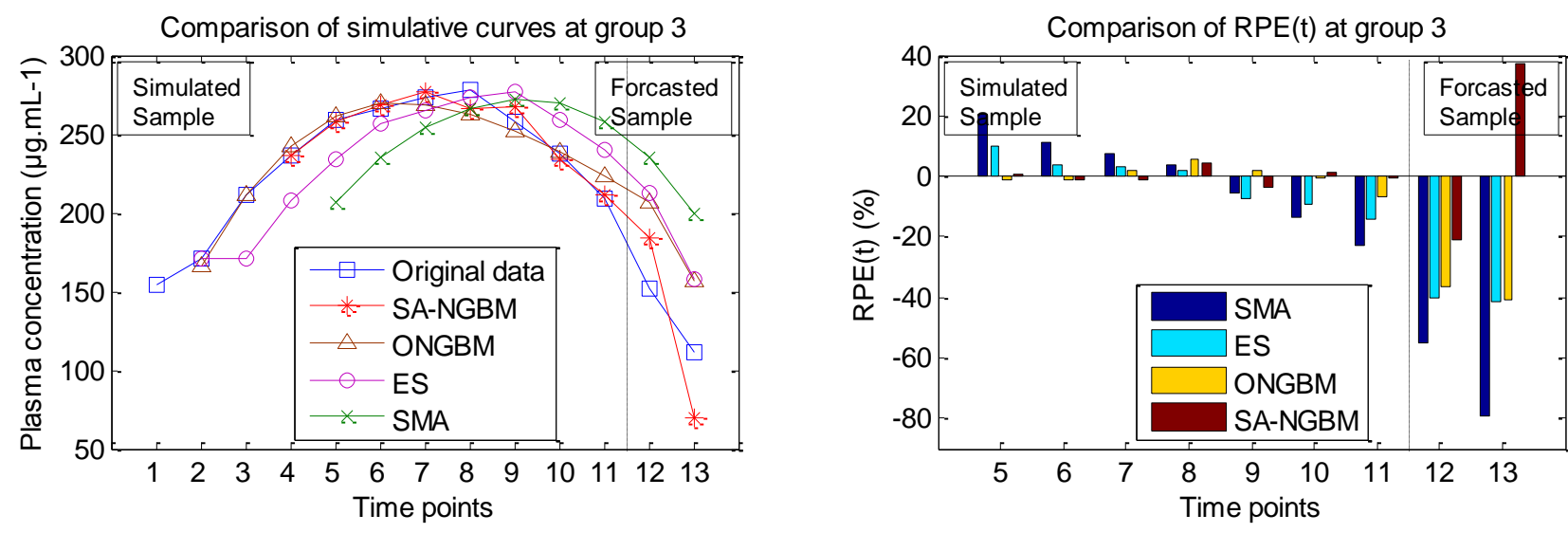

FIGURE 5 Comparison among original and simulative curves of different prediction models of group 3.
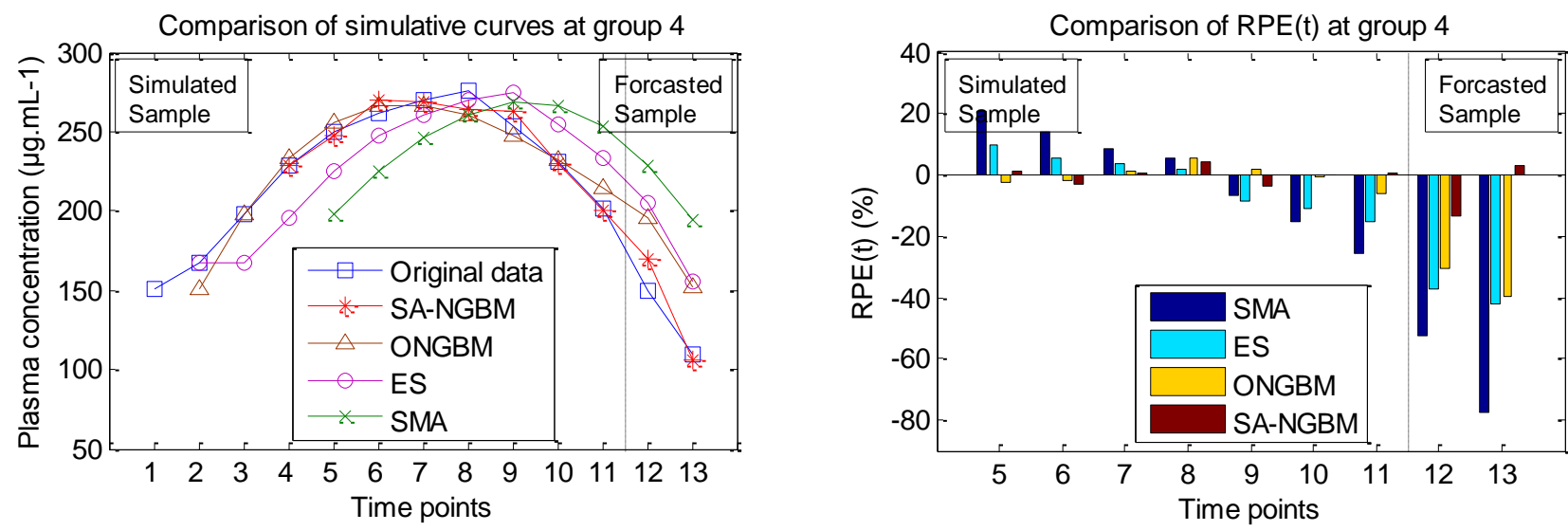

FIGURE 6 Comparison among original and simulative curves of different prediction models of group 4.

In addition, from the comparison of results across accuracy and significance tests, the residual error, posterior variance, correlation degree and error significance of the SA-NGBM were widely superior to that of other prediction models. Thus, according to grey prediction theory $[9,20]$, the proposed SA-NGBM could be used to carry out extrapolation and prediction that could reasonably reflect the decreasing trend of the future plasma concentration of acetylsalicylic acid.

Nevertheless, in real-life situations, there exist numerous influence factors involved in plasma concentration. For example, the individual difference between patients, different dosage regimens, the unknown potential drug interactions, the pathophysiological status of patients, and so forth, could all influence plasma concentrations. Therefore, high-quality plasma time-concentration data are difficult to acquire. With the development of clinical drug trials, the systematic population pharmacokinetics can be conducted in the clinical stage of new drugs. Consequently, the high-quality data samples can be obtained for conducting plasma concentration prediction and guiding clinical medication regimens.

\section{Conclusion and future work}

In this paper, we propose a self-memory algorithm-based NGBM model termed SA-NGBM and utilize it to predict the plasma concentration of acetylsalicylic acid. The model combines the prediction advantages of the self-memory algorithm and the traditional NGBM. SA could be useful for catching the individual stochastic fluctuations existing in nonlinear systems due to uncertain external disturbances. The system's self-memorization equation of the SA-NGBM contains a multiple-time-point initial field instead of an initial value determined from a single time point. Consequently, it overcomes the limitations of being sensitive to the initial value used in the traditional NGBM and possesses more robust predictions. The empirical results indicated that the new model can take full advantage of the historical statistical information and capture tightly the nonlinear system's tendency of 
integral evolution and individual fluctuations. It is worth popularizing and applying to forecast other single-peaked time sequences with similar sample sizes.

Plasma concentration prediction and monitoring for drugs are important to guide the rational use of drugs as clinical medications. Accurate predictions are conducive to reduce the adverse reactions of patients, and are expected to provide more convenience for drug developers. In clinical environments, the original plasma time-concentration data distributions often present downward trends, such as intravenous administration, or the single-peaked types, such as extravascular administration. Nevertheless, many plasma time-concentration data that are required by conventional pharmacokinetics cannot be acquired easily. Under the condition of small sample sizes, the clinical plasma concentration sequence characteristics of single-peaked series can also be predicted using analogical modeling. Once the accuracy and significance test has been passed, the model can be used for extrapolation forecasting. Accordingly, through the prediction method proposed in this paper, similar small sample-size, single-peaked, plasma concentration series of other drugs can be predicted by analogy. Patients can be individually tracked and treated, allowing for drug dosage, physical metabolism and other drug guidance programs to be obtained more scientifically and accurately. By means of the combination of computer algorithms and clinical pharmacology, data-mining methods can be used to improve human health and have good development prospects.

However, the existing research on the SA-NGBM is not comprehensive in this paper. Problems such as the intelligent optimization algorithms of the retrospective order have not been discussed. Additionally, the SA-NGBM is a univariable nonlinear grey Bernoulli model and cannot reflect the effect of relevant factors in multivariable nonlinear systems with interacting relationships. In addition, new plasma time-concentration data will continually enter into the original systems as a result of the periodical monitoring when considering the time-concentration curve for repeated administrations. The metabolism model based on the new information priority principle may be introduced into the SA-NGBM. Therefore, there remain some solutions that should be explored for these problems.

\section{Acknowledgments}

The authors are grateful to the editors and the anonymous referees for their constructive comments and suggestions that helped to improve the clarity and completeness of this paper. This work was supported by a Marie Curie International Incoming Fellowship within the 7th European Community Framework Programme (No. FP7-PIIF-GA-2013-629051), the National Natural Science Foundation of China (Nos. 71603135 and 71871084), the Humanistic and Social Science Youth Foundation of Ministry of Education of China (Nos. 15YJC630048 and 18YJC630043), the Funding of Nantong Science and Technology Program (No. MS12015013) and the Jiangsu Government Scholarship for Overseas Studies.

\section{References}

[1] J.H. Yang, Physiological-atrium model, J. Biomath. 14 (4) (1999) 440-444.

[2] P. Neelamegam, A. Jamaludeen, A. Rajendran, Prediction of calcium concentration in human blood serum using an artificial neural network, Measurement 44 (2011) 312-319.

[3] A. Wiesel, O. Bibi, A. Globerson, Time varying autoregressive moving average models for covariane estimation, IEEE Trans. Signal Process. 61 (2013) 2791-2801.

[4] T. Matsubara, J. Morimoto, Bilinear modeling of EMG signals to extract user-independent features for multiuser myoelectric interface, IEEE Trans. Biomed. Engin. 60 (2013) 2205-2213.

[5] G. Li, C. Wen, W. Zheng, Y. Chen, Identification of a class of nonlinear autoregressive models with exogenous inputs based on kernel machines, IEEE Trans. Signal Process. 59 (5) (2011) 2146-2159.

[6] L.A. Zadeh, Fuzzy sets, Inform. Control 8 (1965) 338-353.

[7] Z. Pawlak, Rough sets, Int. J. Inform. Comp. Sci. 11 (1982) 341-356.

[8] J.L. Deng, Control problems of grey systems, Syst. Control Lett. 1 (5) (1982) 288-294.

[9] S.F. Liu, Grey System Theory and Its Application (8th ed), Beijing: Science Press, 2016.

[10] N.M. Xie, S.F. Liu, Y.J. Yang, C.Q. Yuan, On novel grey forecasting model based on non-homogeneous 
index sequence, Appl. Math. Model. 37 (7) (2013) 5059-5068.

[11] L. Wu, S. Liu, Y. Yang, Grey double exponential smoothing model and its application on pig price forecasting in China, Appl. Soft Comput. 39 (2016) 117-123.

[12] J. Ye, Y. Dang, B. Li, Grey-Markov prediction model based on background value optimization and central-point triangular whitenization weight function, Commun. Nonlinear Sci. Numer. Simul. 54 (2018) 320-330.

[13] S. Ding, K. Hipel, Y.G. Dang, Forecasting China's electricity consumption using a new grey prediction model, Eng. 149 (2018) 314-328.

[14] B. Zeng, H.M. Duan, Y. Bai, W. Meng, Forecasting the output of shale gas in China using an unbiased grey model and weakening buffer operator, Eng. 151 (2018) 238-249.

[15] L.F. Wu, S.F. Liu, Y.J. Yang, L.H. Ma, H.X. Liu, Multi-variable weakening buffer operator and its application, Inform. Sci. 339 (2016) 98-107.

[16] H. Guo, X.P. Xiao, J. Forrest, A research on a comprehensive adaptive grey prediction model CAGM(1,N), Appl. Math. Comput. 225 (1) (2013) 216-227.

[17] Y.T. Lee, Structure activity relationship analysis of phenolic acid phenethyl esters on oral and human breast cancers: The grey $\mathrm{GM}(0, \mathrm{~N})$ approach, Comput. Biol. Med. 41 (2011) 506-511.

[18] L.P. Zhang, Y.L. Zheng, K. Wang, X.L. Zhang, Y.J. Zheng, An optimized Nash nonlinear grey Bernoulli model based on particle swarm optimization and its application in prediction for the incidence of Hepatitis B in Xinjiang, China, Comput. Biol. Med. 49 (2014) 67-73.

[19] X.J. Shen, L.M. Ou, X.J. Chen, X. Zhang, X.R. Tan, The application of the grey disaster model to forecast epidemic peaks of typhoid and paratyphoid fever in China, PLoS One 8 (4) (2013) e60601.

[20] S.F. Liu, Y.J. Yang, J. Forrest, Grey data analysis: methods, Models and Applications, New York: Springer, 2017.

[21] N.M. Xie, S.F. Liu, Discrete grey forecasting model and its optimization, Appl. Math. Model. 33 (2) (2009) 1173-1186.

[22] L.F. Wu, S.F. Liu, L.G. Yao, S.L. Yan, D.L. Liu, Grey system model with the fractional order accumulation, Commun. Nonlinear Sci. Numer. Simul. 18 (7) (2013) 1775-1785.

[23] L.F. Wu, S.F. Liu, Z.G. Fang, H.Y. Xu, Properties of the GM(1,1) with fractional order accumulation, Appl. Math. Comput. 252 (2015) 287-293.

[24] C.I. Chen. Application of the novel nonlinear grey Bernoulli model for forecasting unemployment rate, Chaos Soliton. Fract. 37 (1) (2008) 278-287.

[25] C.I. Chen, H.L. Chen, S.P. Chen, Forecasting of foreign exchange rates of Taiwan's major trading partners by novel nonlinear Grey Bernoulli model NGBM(1,1), Commun. Nonlinear Sci. Numer. Simul. 13 (6) (2008) 1194-1204.

[26] H.X. Cao, Self-memorization equation in atmospheric motion, Sci. Chin. Ser. B 36 (7) (1993) 845-855.

[27] N. Phienwej, S. Thepparak, P.H. Giao, Prediction of differential settlement of buildings induced by land subsidence from deep well pumping, Geotech. Engin. 36 (1) (2005) 69-76.

[28] X.P. Liu, X.B. Xie, Y.Z. Luo, Self-memorization model of dynamic system for predicting nonlinear displacement of slopes, Chin. J. Geotech. Engin. 32 (2010) 1535-1542.

[29] W. Wang, J.Y. Su, B.W. Hou, J. Tian, D.H. Ma, Dynamic prediction of building subsidence deformation with data-based mechanistic self-memory model, Chin. Sci. Bull. 57 (23) (2012) 2171-2176.

[30] X.H. Fan, Y. Zhang, A novel self-memory grey model, Syst. Eng. Theory Pract. 23 (2003) 114-117.

[31] X.D. Chen, J. Xia, Q. Xu, Differential hydrological grey model (DHGM) with self-memory function and its application to flood forecasting, Sci. Chin. Ser. E: Tech. Sci. 39 (2) (2009) 341-350.

[32] X.J. Guo, S.F. Liu, Z.G. Fang, Self-memory prediction model of interval grey number based on grey degree of compound grey number, Syst. Eng. Electron. 36 (6) (2014) 1124-1129.

[33] D.G. Zill, M.R. Cullen, Advanced engineering mathematics, 3rd Edition, Massachusetts: Jones and Bartlett, 2006.

[34] Z.X. Wang, Y.G. Dang, S.F. Liu, Z.W. Lian, Solution of GM(1,1) power model and its properties, Syst. Eng. Electron. 31 (10) (2009) 2380-2383.

[35] Z.X. Wang, K.W. Hipel, Q. Wang, S.W. He, An optimized $\operatorname{NGBM}(1,1)$ model for forecasting the qualified discharge rate of industrial wastewater in China, Appl. Math. Model. 35 (12) (2011) 5524-5532.

[36] Z.X. Wang, Y.G. Dang, J.J. Zhao, Optimized GM(1,1) power model and its application, Syst. Eng. Theory 
Pract. 32 (9) (2012) 1973-1978.

[37] Z.X. Wang, An optimized Nash nonlinear grey Bernoulli model for forecasting the main economic indices of high technology enterprises in China, Comput. Ind. Eng. 64 (3) (2013) 780-787.

[38] H.H. Yan, Z.H. Zhu, L.M. Liu, Q.X. Fang, G. Wang, H.J. Zhao, Prediction of lasma concentration based on wavelet neural network time series model, Chin. J. Mod. Appl. Pharm. 33 (11) (2016) 1417-1422.

[39] B. Billah, M.L. King, R.D. Snyder, A.B. Koehler, Exponential smoothing model selection for forecasting, Int. J. Forecast. 22 (2) (2006) 239-247.

[40] F.R. Johnston, J.E. Boyland, M. Meadows, E. Shale, Some properties of a simple moving average when applied to forecasting a time series, J. Oper. Res. Soc. 50 (1999) 1267-1271. 


\section{Author Biography}

Xiaojun Guo is an Assistant Professor in the School of Science, Nantong University, Nantong, China. He received his M.S. degree in Epidemiology and Health Statistics from Nantong University, Nantong, China, in 2007. And he received his Ph.D. degree in Management Science and Engineering from Nanjing University of Aeronautics and Astronautics, Nanjing, China, in 2015. His current research interests include grey systems theory, small sample time series analysis, mathematical biology and epidemiology.

Sifeng Liu is a Professor in the College of Economics and Management, Nanjing University of Aeronautics and Astronautics, Nanjing, China. He received his Ph.D. degree in Grey Systems Theory from Huazhong University of Science and Technology, Wuhan, China, in 1998. He is the Chairman of IEEE Grey System Society and Vice Chairman of IEEE SMC China (Beijing) Branch. His current research interests include grey systems theory, management science and project management.

Yingjie Yang is a Professor in the Centre for Computational Intelligence, De Montfort University, UK. He received his Ph.D. degree in Mathematics from Northeastern University, Shenyang, China, in 1994. His current research interests include grey systems theory, management science and decision making. 
Editing Certificate
Click here to download Supplementary Material: Editing Certificate.pdf

(n)

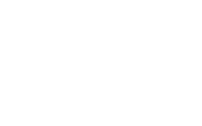

(1)

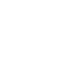
(1)

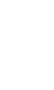
(1) (1)

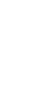

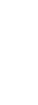

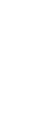
(1)

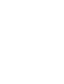

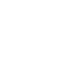

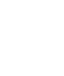

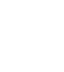
. . . . . . . . 